\title{
Un teorema di esistenza della soluzione del problema di Cauchy nella classe delle funzioni assolutamente continue nelle singole variabili $(*)(* *)$.
}

\author{
Maria Grazia Cazzani Nieri (Pavia)
}

Sunto. - È dimostrato un teorema di esistenza per il problema di Cauchy relativo ai sistemi semilineari di tipo iperbolico in due variabiti indipendenti

$$
\sum_{i=1}^{m} a_{i j}(x, y)\left\{p_{j}+e_{i}(x, y) q_{i}\right\}=f_{i}\left(x, y, z_{1}, \ldots, z_{m}\right), \quad(i=1, \ldots, m),
$$

in una nuova, ampia classe funzionale: la soluziane è ricercata nel campo funzionale costituito dalle m-ple di junzioni $z_{j}(x, y),(j=1, \ldots, m)$, le quali sono assolutamente continue nelle singole variabili $x, y$ separatamente e soddisfano il sistema (I) quasi ovunqu. Bono costruiti inottre due controesempi, che giustificano le ipotesi introdotte, ed d dimostrato un lemma, relativo alle equazioni differenziali ordinarie, utilizzato nella dimostrazione del teorema di esistenza.

\section{Introduzione.}

Il problema di Cauchy relativo ai sistemi di equazioni a derivate parziali quasi lineari di tipo iperbolico in più variabili indipendenti $x, y_{1}, \ldots, y_{r}$ è stato ampiamente considerato e sono state dimostrate l'esistenza e l'unicità della soluzione (in senso generalizzato) nel campo delle funzioni continue, le quali inoltre sono assolutamente continue in $x$ e lipschitziane nel complesso delle variabili $\left.y_{1}, \ldots, y_{r}{ }^{1}\right)$.

Nel presente lavoro consideriamo il problema di Cauchy per i sistemi semilineari di tipo iperbolico in due variabili indipendenti in forma caratteristica

$$
\sum_{j=1}^{m} a_{i j}(x, y)\left\{p_{j}+\varrho_{i}(x, y) q_{j}\right\}=f_{i}\left(x, y, z_{1}, \ldots, z_{m}\right) \quad(i=1, \ldots, m)
$$

(*) Entrata in Redazione il 9 marzo 1976.

(**) Lavoro eseguito nell'ambito del G.N.A.F.A. del C.N.R. I risultati ottenuti nol presente lavoro sono stati comunicati a Cagliari, in occasione del $\mathrm{X}$ Congresso U.M.I., 22-28 settembre 1975 .

(i) M. CINQUin I CIbrario, Teoremi d'esistenza per sistemi semilineari di equazioni a derivate parziali in più variabili indipendenti, Annali di Mat., S. IV, 68 (1965), pp. 119-160.

M. CINQUin CIBraRIo, Teoremi di esistenza per sistemi di equazioni quasi lineari a derivate parziali in più variabili indipendenti, Annali di Mat., S. IV, 75 (1967), pp. 1-46.

M. Crnquinr Crbrario, Teoremi di unieità per sistemi di equazioni a derivate parziali in piu variabili indipendenti, Annali di Mat., S. IV, 48 (1959), pp. 103-134. 
in una nuova e più ampia classe funzionale: la soluzione

$$
z_{1}(x, y), \quad z_{2}(x, y), \ldots, z_{m}(x, y)
$$

è ricereata nella classe delle funzioni assolutamente continue rispetto alle singole variabili $x, y$ separatamente.

E stabilito un teorema di esistenza relativo al problema di Cauchy per il sistema (I).

Le funzioni $a_{i j}(\ldots)$ sono supposte continue, assolutamente continue in $x$ e lipschitziane in $y$, le funzioni $\varrho_{i}(\ldots), f_{i}(\ldots)$ sono quasi continue in $x$ e soddisfano nel complesso delle rimanenti variabili condizioni del tipo di Carathéodory; inoltre si suppone che il campo di definizione $D_{0}$ delle funzioni $\varrho_{i}(x, y)$ sia decomponibile in un numero finito di campi, in ciascuno dei quali, a meno di un insieme di misura nulla (precisamente per quasi tutti gli $x$ ) le funzioni $\varrho_{i}(x, y),(i=1, \ldots, m), \varrho_{i}(x, y)-\varrho_{h}(x, y)$, $(i, h=1, \ldots, m ; i \neq h)$, non cambino segno.

Si dimostra l'esistenza di una $m$-pla di funzioni $z_{1}(x, y), \ldots, z_{m}(x, y)$, assolutamente continue rispetto alle singole variabili, le quali soddisfano il sistema (I) quasi ovunque nel campo $D_{0}$ e inoltre, nei punti di $D_{0}$ di ascissa nulla, le condizioni

$$
z_{i}(0, y)=\phi_{i}(y) \quad(i=1, \ldots, m),
$$

essendo $\phi_{i}(y)$ funzioni assolutamente continue assegnate.

Più precisamente le funzioni $z_{i}(x, y),(i=1, \ldots, m)$, risultano equiassolutamente continue in $x$ al variare di $y$ e equiassolutamente continue in $y$ al variare di $x$, nel senso precisato nell'enunciato del teorema.

Nella dimostrazione, considerate le caratteristiche del sistema (I), è introdotto un sistema di equazioni integrali (cfr. le (10)) : per tale sistema è dimostrata l'esistenza della soluzione $z_{i}(x, y),(i=1, \ldots, m)$, la quale risulta soluzione quasi ovunque nel campo $D_{0}$ del sistema (I) sotto le condizioni (II).

Le funzioni $z_{i}(x, y),(i=1, \ldots, m)$, risultano assolutamente continue su ogni curva caratteristica fissata.

Nel $\$ 2$ si giustificano in un certo senso le ipotesi relative al segno delle funzioni $\varrho_{i}(\ldots), \varrho_{i}(\ldots)-\varrho_{h}(\ldots)$, mostrando con un esempio che, se una funzione $\varrho_{i}(\ldots)$ cambia segno infinite volte al variare di $x$, la soluzione del sistema di equazioni integrali, nel campo funzionale precisato, può mancare. Pertanto, se non si aggiunge un'ipotesi sulle funzioni $g_{i}(\ldots)$, per esempio sul loro segno, il teorema di esistenza, almeno per il sistema di equazioni integrali, non sussiste.

Con un ulteriore esempio è messo in luce che, se $\varrho_{i}(\ldots)-\varrho_{h}(\ldots)$, per una coppia fissata di valori $i, h$, cambia segno infinite volte al variare di $x$, le funzioni $z_{i}(x, y)$, $(i=1, \ldots, m)$, soluzione del particolare sistema di equazioni integrali considerato, sono sì assolutamente continue rispetto alle singole variabili, ma, considerate lungo le curve caratteristiche, non sono assolutamente continue, precisamente non sono neppure a variazione limitata. In assenza di quest'ultima proprietà non sembra 
possibile affrontare la costruzione di una successione di funzioni approssimanti la soluzione del sistema di equazioni integrali, nel suddetto campo funzionale.

Inoltre alla fine del $\S 2$ (cf. n. 10) è dimostrato un lemma, riguardante le equazioni differenziali ordinarie, che è utilizzato nella dimostrazione del teorema di esistenza del $\S 1$.

\section{§1.}

\section{1. - Teorema di esistenza.}

Sia $M(x)$ una funzione quasi continua, non negativa e integrabile ${ }^{\left({ }^{b i s}\right)}$ nell'intervallo $\left(0, a_{0}\right)$ e sia $b_{0}$ un numero positivo tale che per $0 \leqslant x<a_{0}$ sia $\int_{0}^{x} M(t) d t<b_{0}$,
mentre per $x=a_{0}$ può anche valere l'uguaglianza.

Sia $D_{0}$ il campo definitivo dalle diseguaglianze

$$
D_{0}: \quad 0 \leqslant x \leqslant a_{0}, \quad-b_{0}+\int_{0}^{x} M(t) d t \leqslant y \leqslant b_{0}-\int_{0}^{x} M(t) d t .
$$

Siano $a_{i j}(x, y),(i, j=1, \ldots, m)$, funzioni continue in $D_{0}$, le quali in corrispondenza ad ogni $x d i\left(0, a_{0}\right)$ siano lipsehitziane in $y$ nell'intervallo $I_{x}\left(-b_{0}+\int_{0}^{x} M(t) d t, b_{0}-\int_{0}^{x} M(t) d t\right)$ e in corrispondenza ad ogni $y d i\left(-b_{0}, b_{0}\right)$ siano assolutamente continue in $x$ nell'intervallo $I_{y}$ dei valori $x$, tali ohe $i$ punti $(x, y)$ appartengano al campo $D_{0}$; inolle esista una funzione $\mu(x),\left(0 \leqslant x \leqslant a_{0}\right)$, non negativa, quasi continua e integrabile, tale che sia

$$
\left|a_{i j}\left(x^{\prime}, y\right)-a_{i j}\left(x^{\prime \prime}, y\right)\right| \leqslant\left|\int_{x^{\prime}}^{x^{\prime \prime}} \mu(x) d x\right|
$$

per ogni coppia di punti $\left(x^{\prime}, y\right),\left(x^{\prime \prime}, y\right)$ del campo $D_{0} ;$ sia $\Lambda$ una costante non negativa tale che per tutte le coppie di punti $(x, y),(x, \bar{y})$ di $D_{0}$ sia

$$
\left|a_{i j}(x, y)-a_{i j}(x, \bar{y})\right| \leqslant A|y-\bar{y}|
$$

Detto $A$ il determinante delle funzioni $a_{i j}(\ldots),(i, j=1, \ldots, m)$, in tutto il campo $D_{0}$ sia

$$
A=1
$$

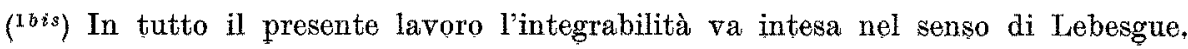


Siano $\varrho_{i}(x, y),(i=1, \ldots, m)$, funzioni definite in $D_{0}$, quasi continue in $x$ su ogni intervallo $I_{y}, e$, in corrispondenza ad ogni $x$ di $\left(0, a_{0}\right)$, continue in $y$ nell'intervallo $I_{x}$; per quasi tuti gli $x$ di $\left(0, a_{0}\right)$ sia

$$
\left|\varrho_{i}(x, y)\right| \leqslant M(x) \quad(i=1, \ldots, m)
$$

per ogni y di $I_{x}$; esista una funzione $L(x)$ in $\left(0, a_{0}\right)$, quasi continua, non negativa $e$ integrabile, tale che per quasi tutti gli $x$ di $\left(0, a_{0}\right)$ sia

$$
\left|\varrho_{i}(x, y)-\varrho_{i}(x, \bar{y})\right| \leqslant L(x)|y-\bar{y}| \quad(i=1, \ldots, m)
$$

per ogni coppia $y, \breve{y} d i I_{2}$.

Siano $f_{i}\left(x, y, z_{1}, \ldots, z_{m}\right),(i=1, \ldots, m)$, funzioni definite nel campo

$C_{c o}: \quad 0 \leqslant x \leqslant a_{0}, \quad-b_{0}+\int_{0}^{x} M(t) d t \leqslant y \leqslant b_{0}-\int_{0}^{\infty} M(t) d t, \quad-\infty<z_{j}<+\infty, \quad(j=1, \ldots, m)$,

quasi continue rispetto a $w$ in $I_{y}$ per ogni $(n+1)$-pla $\left(y, z_{1}, \ldots, z_{n n}\right)$ fissata, e, per ogni $x$ fissato in $\left(0, a_{0}\right)$, continue nel complesso delle variabili $y, z_{1}, \ldots, z_{m}$ nel campo

$\Delta_{x}: \quad-b_{0}+\int_{0}^{x} M(t) d t \leqslant y \leqslant b_{0}-\int_{0}^{x} M(t) d t, \quad-\infty<z_{j}<+\infty(j=1, \ldots, m)$

esistano due funzioni $N(x), L_{1}(x)$ quasi continue, non negative e integrabili in $\left(0, a_{0}\right)$, tali che per quasi tutti gli $x$ di $\left(0, a_{0}\right)$ sia

$$
\left|f_{i}\left(x, y, z_{1}, \ldots, z_{m}\right)\right| \leqslant N(x), \quad(i=1, \ldots, m)
$$

per ogni $(m+1)$-pla $\left(y, z_{1}, \ldots, z_{m}\right)$ del campo $\Delta_{x}, e$

(5) $\left|f_{i}\left(x, y, z_{1}, \ldots, z_{m}\right)-f_{i}\left(x, \bar{y}, \bar{z}_{1}, \ldots, \bar{z}_{m}\right)\right| \leqslant L_{1}(x)\left\{|y-\bar{y}|+\sum_{j=1}^{n}\left|z_{j}-\bar{z}_{j}\right|\right\} \quad(i=1, \ldots, m)$

per ogni coppia di $(m+1)$-ple $\left(y, z_{1}, \ldots, z_{m}\right),\left(\bar{y}, \bar{z}_{1}, \ldots, \bar{z}_{m}\right) d i \Delta_{x}$.

Inottre l'intervallo $\left(0, a_{0}\right)$ sia decomponibile in un numero finito di intervalli, in eiascuno dei quali per quasi tutti gli $x$ vale, per ogni $y$ di $I_{x}$, una delle relazioni

$$
\varrho_{i}(x, y) \geqslant 0 \quad \text { ovvero } \quad \varrho_{i}(x, y) \leqslant 0
$$


per ogni $i$ compreso tra 1 e m, e per ogni coppia di valori distinti $i, h,(i, h=1, \ldots, m$, $i \neq h$ ), una delle relazioni

$$
\varrho_{i}(x, y) \leqslant \varrho_{k}(x, y) \quad \text { ovvero } \quad \varrho_{i}(x, y) \geqslant \varrho_{k}(x, y) .
$$

Siano infine $\phi_{1}(y), \phi_{2}(y), \ldots, \phi_{m}(y)$ funzioni assolutamente continue in $\left(-b_{0}, b_{0}\right)$. In queste ipotesi esiste una m-pla di funzioni

$$
z_{1}(x, y), z_{2}(x, y), \ldots, z_{m}(x, y)
$$

definite nel campo

$$
D_{0}: \quad 0 \leqslant x \leqslant a_{0}, \quad-b_{0}+\int_{0}^{x} M(t) d t \leqslant y \leqslant b_{0}-\int_{0}^{x} M(t) d t
$$

le quali in corrispondenza ad ogni $y$ di $\left(-b_{0}, b_{0}\right)$ sono assolutamente continue in $x$ nell'intervallo $I_{y}$, in modo uniforme rispetto a $y$, in corrispondenza ad ogni $x$ di $\left(0, a_{0}\right)$ sono assolutamente continue in y nell'intervallo $I_{x}$, in modo uniforme rispetto a $x\left(^{2}\right)$, soddisfano quasi ovunque in $D_{0}$ il sistema di equazioni a derivate parziali di tipo iperbolico semilineare in due variabili indipendenti (in forma caratteristica)

$$
\sum_{i=1}^{m} a_{i j}(x, y)\left\{p_{j}+\varrho_{i}(x, y) q_{j}\right\}=f_{i}\left(x, y, z_{1}, \ldots, z_{m}\right), \quad(i=1, \ldots, m)
$$

(dove $\left.p_{j}=\partial z_{j} / \partial x, q_{j}=\partial z_{j} / \partial y\right)$ e inoltre in $\left(-b_{0}, b_{0}\right)$ le condizioni

$$
z_{i}(0, y)=\phi_{i}(y) \quad(i=1, \ldots, m) .
$$

Premettiamo alla dimostrazione, che è sviluppata nei nn. 5, 6, alcune considerazioni preliminari (efr. nn. 2,3,4).

\section{2. - Curve caratteristiche.}

Definiamo nel campo

$D_{\infty}$ :

$$
0 \leqslant x \leqslant a_{0}, \quad-\infty<y<+\infty
$$

$\left({ }^{2}\right)$ Sono assolutamente continue per esempio rispetto a $y$ in modo uniforme rispetto a $x$ nel senso che, preso $\varepsilon>0$, si può determinare un numero $\delta>0$, tale che, se $\left(y_{1}^{\prime}, y_{1}^{\prime \prime}\right), \ldots,\left(y_{r}^{\prime}, y_{r}^{\prime \prime}\right)$ sono $r$ intervalli di $I_{x}$ non sorrapposti a due a due di ampiezza complessiva minore di $\delta$, per ogni $x$ di $\left(0, a_{0}\right)$ sia

$$
\sum_{k=1}^{r}\left|z_{i}\left(x, y_{k}^{\prime}\right)-z_{i}\left(x, y_{k}^{\prime \prime}\right)\right|<\varepsilon, \quad(i=1, \ldots, m) .
$$


le funzioni $\tilde{\varrho}_{i}(x, y)$ nel seguente modo:

$$
\begin{aligned}
& \tilde{\varrho}_{i}(x, y)=\varrho_{i}(x, y) \quad \text { nel campo } D_{0} ; \\
& \tilde{\varrho}_{i}(x, y)=\varrho_{i}\left(x, b_{0}-\int_{0}^{x} M(t) d t\right) \text { per } y>b_{0}-\int_{0}^{x} M(t) d t ; \\
& \tilde{\varrho}_{i}(x, y)=\varrho_{i}\left(x,-b_{0}+\int_{0}^{x} M(t) d t\right) \text { per } y<-b_{0}+\int_{0}^{x} M(t) d t, \quad(i=1, \ldots, m) .
\end{aligned}
$$

Nel campo $D_{\infty}$, le funzioni $\tilde{\varrho}_{i}(x, y)$ soddisfano evidentemente le $(2)$ per quasi tutti gli $x$ di $\left(0, a_{0}\right)$ e per ogni $y$, le (3) per quasi tutti gli $x$ di $\left(0, a_{0}\right)$ e per ogni coppia $y, \bar{y}$.

Siano

$$
Y=g_{i}(X ; x, y) \quad(i=1, \ldots, m)
$$

le funzioni definite dalle equazioni integrali

$$
g_{i}(X ; x, y)=y+\int_{x}^{x} \tilde{\varrho}_{i}\left(t, g_{i}(t ; x, y)\right) d t
$$

nel campo

$$
\Delta_{\infty}: \quad 0 \leqslant X \leqslant a_{0}, \quad 0 \leqslant x \leqslant a_{0}, \quad-\infty<y<+\infty .
$$

Valgono i teoremi di esistenza e unicità di Carathéodory ${ }^{(26 i s)}$ per l'equazione (8).

Le funzioni $g_{i}(X ; x, y),(i=1, \ldots, m)$, sono assolutamente continue in $X$ in modo uniforme rispetto alle rimanenti variabili $x, y$, assolutamente continue in $x$ in modo uniforme rispetto a $X, y$, lipsehitziane in $y$ con costante di Lipschitz indipendente da $x, X\left({ }^{3}\right)$. Risulta

$$
\left|g_{i}(X ; x, y)-g_{i}(X ; x \bar{y})\right| \leqslant \lambda_{1}|y-\bar{y}|
$$

$\operatorname{con} \lambda_{1}=\exp \left[\int_{0}^{a_{0}} L(t) d t\right]$, per ogni coppia di punti $(X ; x, y),(X, x, \bar{y})$ di $\Delta_{\infty}$.

Inoltre, in virtù del citato teorema di unicità, le funzioni $g_{i}(X ; x, y),(i=1, \ldots, m)$, risultano crescenti in $y$; in ciascuna delle strisce di $D_{\infty}$, in cui vale una determinata delle (6), sono monotone anche rispetto a $X, x$, e precisamente non decrescenti in $X$ e non crescenti in $x$ se vale la prima delle (6), non crescenti in $X$ e non decrescenti in $x$ se vale la seconda delle (6).

${ }^{\left({ }^{b i s}\right)}$ Cfr. C. CARATHÉodoRY, Vorlesungen über reelle Funktionen, Leipzig (1918), Kap. XI, pp. $665-688$.

${ }^{(3)}$ Cfr. M. Cinquini-Cibrario, Sistemi di equazioni a derivate parziali in piu variabili indipendenti, Annali di Mat., (IV), 44 (1957), pp. 357-418, § 1, n. 1, 
Se $(x, y)$ appartiene a $D_{0}$, per ogni $X$ di $(0, x)$ il punto $\left(X, g_{i}(X ; x, y)\right)$ appartiene a $D_{0}$.

Pertanto in corrispondenza ad ogni $(x, y)$ di $D_{0}$, per ogni $X$ di $(0, x) \grave{e}\left({ }^{4}\right)$

$$
g_{i}(X ; x, y)=y+\int_{x}^{X} \varrho_{i}\left(t, g_{i}(t ; x, y)\right) d t, \quad(i=1, \ldots, m) .
$$

\section{3. - Un sistema di equazioni integrali.}

a) Il teorema di esistenza del $\mathrm{n} .1$ si può ricondurre ad un teorema di esistenza per un sistema di equazioni integrali.

Nelle ipotesi del $n .1$ sulle funzioni $a_{i j}(\ldots), \varrho_{i}(\ldots), f_{i}(\ldots), \phi_{i}(\ldots)$, esistano infatti $n$ funzioni $z_{1}(x, y), \ldots, z_{m}(x, y)$, assolutamente continue nelle singole variabili $x, y$, le quali soddisfino nel campo $D_{0}$ il sistema di equazioni integrali

$$
\begin{aligned}
& \sum_{j=1}^{m} a_{i j}(x, y) z_{j}(x, y)=\sum_{j=1}^{m} a_{i j}\left(0, g_{i}(0 ; x, y)\right) \phi_{j}\left(g_{i}(0 ; x, y)\right)+ \\
& \quad+\int_{0}^{x}\left[\sum_{j=1}^{m} \frac{d a_{i j}\left(X, g_{i}(X ; x, y)\right)}{d X} z_{j}\left(X, g_{i}(X ; x, y)\right)+\right. \\
& \left.\quad+f_{i}\left(X, g_{i}(X ; x, y), z_{1}\left(X, g_{i}(\ldots)\right), \ldots, z_{m}(\ldots)\right)\right] d X, \quad(i=1, \ldots, m)
\end{aligned}
$$

allora in quasi tutto il campo $D_{0}$ valgono le

$$
\sum_{j=1}^{m} a_{i j}(x, y)\left\{p_{j}(x, y)+\varrho_{i}(x, y) q_{j}(x, y)\right\}=f_{i}\left(x, y, z_{1}(x, y), \ldots, z_{m}(x, y)\right)
$$

e in tutto l'intervallo $\left(-b_{0}, b_{0}\right)$ le

$$
(i=1, \ldots, m)
$$

$$
z_{j}(0, y)=\phi_{j}(y), \quad(j=1, \ldots, m) .
$$

Le (II) sono evidenti: infatti dalle (10) per $x=0$, tenuto conto che è $g_{i}(0 ; 0, y)=y$, si ha

$$
\sum_{j=1}^{m} a_{i j}(0, y) z_{j}(0, y)=\sum_{j=1}^{m} a_{i j}(0, y) \phi_{j}(y)
$$

tenuto conto della $\left(1^{\prime \prime}\right)$, ne seguono le (II) in tutto $\left(-b_{0}, b_{0}\right)$.

$\left({ }^{4}\right)$ Relativamente alla $\left(8^{\prime}\right)$ e alle proprietà di monotonia delle funzioni $g_{i}(\ldots)$ cfr. M. G. Cazzani Nieri, Su un problema misto per un sistema di equazioni a derivate parziali, Annali di Mat., (IV), 77 (1967), pp. 131-178; cfr. p. 137. 
Rileviamo che nelle ipotesi fatte sulle funzioni $a_{i j}(X, Y), \varrho_{i}(\ldots)$, le funzioni $a_{i j}\left(X, g_{i}(X ; x, y)\right)$ sono assolutamente continue in $X$; per quasi tutti gli $X$, tali che il punto $\left(X, g_{i}(X ; x, y)\right)$ appartenga a $D_{0}$, tali funzioni sono derivabili; in corrispondenza ad ogni $x$ fissato in $\left(0, a_{0}\right)$, per quasi tutti gli $y$ di $I_{x}$, per quasi tutti gli $X$, è

$$
\frac{d a_{i j}\left(X, g_{i}(X ; x, y)\right)}{d X}=\frac{\partial a_{i j}(\ldots)}{\partial X}+\varrho_{i}(\ldots) \frac{\partial a_{i j}(\ldots)}{\partial X}, \quad(i, j=1, \ldots, m) .
$$

Per dimostrare che in quasi tutto $D_{0}$ valgono le $(I)$, posto

$$
g_{i}(0 ; x, y)=\eta,
$$

osserviamo che, in virtù del teorema di unicità citato in $\left({ }^{2 b i s}\right)$, è

$$
g_{i}(X ; x, y)=g_{i}(X ; 0, \eta), \quad\left(0 \leqslant X \leqslant a_{0}\right)
$$

e, per ogni $i$, fissato $\eta$, ̀̀

$$
\begin{aligned}
& \sum_{j=1}^{m} a_{i j}\left(x, g_{i}(x ; 0, \eta)\right) z_{j}\left(x, g_{i}(x ; 0, \eta)\right)=\sum_{j=1}^{m} a_{i j}(0, \eta) \phi_{j}(\eta)+ \\
& +\int_{0}^{\infty}\left[\sum_{j=1}^{m} \frac{d a_{i j}\left(X, g_{i}(X ; 0, \eta)\right)}{d X} z_{j}\left(X, g_{i}(X ; 0, \eta)\right)+\right. \\
& +f_{i}\left(X, g_{i}(X ; 0, \eta), z_{1}(. .), \ldots, z_{m}(\ldots)\right) d X
\end{aligned}
$$

per ogni $x$ tale che il punto $\left(x, g_{i}(x ; 0, \eta)\right)$ appartenga al campo $D_{0}$; indichiamo l'intervallo di tali valori di $x$ con $\left(0, \beta_{i}(\eta)\right)$.

Posto

$$
Z_{i}(x, y)=\sum_{j=1}^{m} a_{i j}(x, y) z_{j}(x, y),
$$

in virtù delle $\left(10^{\prime}\right)$ la funzione $Z_{i}\left(x, g_{i}(x ; 0, \eta)\right)$ risulta assolutamente continua in $x$ nell'intervallo $\left(0, \beta_{i}(\eta)\right)$, e in quasi tutto $\left(0, \beta_{i}(\eta)\right)$ esiste finita la sua derivata.

In quasi tutto $\left(^{(5)} D_{0}\right.$ è

$$
\begin{aligned}
& \frac{\partial Z_{i}(x, y)}{\partial x}=\sum_{j=1}^{m} \frac{\partial a_{i j}(x, y)}{\partial x} z_{j}(x, y)+\sum_{j=1}^{m} a_{i, j}(x, y) \frac{\partial z_{j}(x, y)}{\partial x}, \\
& \frac{\partial Z_{i}(x, y)}{\partial y}=\sum_{j=1}^{m} \frac{\partial a_{i j}(x, y)}{\partial y} z_{j}(x, y)+\sum_{j=1}^{m} a_{i j}(x, y) \frac{\partial z_{j}(x, y)}{\partial y} ;
\end{aligned}
$$

in virtù della corrispondenza biunivoca che la funzione

$$
y=g_{i}(x ; 0, \eta)
$$

$\left(^{5}\right)$ L'insieme dei punti di $D_{0}$ in eui esistono le derivate parziali di $Z_{i}(x, y)$ è misurabile e ha la stessa misura di $D_{0}$. Cfr. 1.c. in $\left({ }^{2}\right)$, Kap. XI, $§ 557$, Satz 1 ; \$ 559, Satz 3. 
pone tra i punti $(x, y)$ del campo $D_{0}$ e i punti $(x, \eta)$ del campo, corrispondente nel piano $x, \eta$, che chiamiamo $\Delta_{0}$, nella quale ad un insieme di misura nulla di $D_{0}$ corrisponde un insieme di misura di $\Delta_{0}$ (e viceversa) $\left({ }^{6}\right)$, le derivate parziali $\partial Z_{i} / \partial x$, $\partial Z_{i} / \partial y$ della funzione $Z_{i}(x, y)$ esistono in quasi tutto $\Delta_{0}$; quindi in corrispondenza a quasi tutti gli $\eta$ di $\left(-b_{0}, b_{0}\right)$ esistono per quasi tutti gli $x$ di $\left(0, \beta_{i}(\eta)\right)$. Allora, per un teorema di derivazione di funzione composta ( ${ }^{(7)}$, per quasi tutti gli $\eta$ di $\left(-b_{0}, b_{0}\right)$ è

$$
\frac{d Z_{i}\left(x, g_{i}(x ; 0, \eta)\right)}{d x}=\frac{\partial Z_{i}\left(x, g_{i}(x ; 0, \eta)\right)}{\partial x}+\varrho_{i}\left(x, g_{i}(x ; 0, \eta)\right) \frac{\partial Z_{i}\left(x, g_{i}(x ; 0, \eta)\right)}{\partial y}
$$

in quasi tutto $\left(0, \beta_{i}(\eta)\right)$, e quindi, tenuto conto delle $\left(10^{\prime}\right),(11)$,

$$
\begin{aligned}
\frac{\partial Z_{i}\left(x, g_{i}(x ; 0, \eta)\right)}{\partial x}+\varrho_{i}\left(x, g_{i}(\ldots)\right) \frac{\partial Z_{i}\left(x, g_{i}(\ldots)\right)}{\partial y}= \\
\quad=\sum_{j=1}^{m}\left[\frac{\partial a_{i j}\left(x, g_{i}(\ldots)\right)}{\partial x}+\varrho_{i}(\ldots) \frac{\partial a_{i j}(\ldots)}{\partial y}\right] z_{j}(\ldots)+f_{i}(\ldots):
\end{aligned}
$$

tenuto conto delle (12) in quasi tutto $A_{0}$ si ha

$$
\begin{aligned}
\sum_{j=1}^{m} a_{i j}\left(x, g_{i}(x ; 0, \eta)\right)\left\{\frac{\partial z_{i}\left(x, g_{i}(x ; 0, \eta)\right)}{\partial x}+\right. & \left.\varrho_{i}(\ldots) \frac{\partial z_{j}(\ldots)}{\partial y}\right\}= \\
& =f_{i}\left(x, g_{i}(x ; 0, \eta), z_{1}\left(x, g_{i}(x ; 0, \eta)\right), \ldots, z_{m}(\ldots)\right),
\end{aligned}
$$

vale a dire in quasi tutto (cf. $\left.\left({ }^{6}\right)\right) D_{0} \grave{e}$

$$
\begin{aligned}
\sum_{j=1}^{m} a_{i j}(x, y)\left\{\frac{\partial z_{j}(x, y)}{\partial x}+\varrho_{i}(x, y)\right. & \left.\frac{\partial z_{j}(x, y)}{\partial y}\right\}= \\
& =f_{i}\left(x, y, z_{1}(x, y), \ldots, z_{m}(\ldots)\right), \quad(i=1, \ldots, m) .
\end{aligned}
$$

Osservazione. - Osserviamo che, nel dimostrare la validità delle (I) in quasi tutto il campo $D_{0}$, interviene soltanto l'assoluta continuità in $x$ delle funzioni $Z_{i}\left(x, g_{i}(x ; 0, \eta)\right), \quad(i=1, \ldots, m), \mathrm{e}$ non delle singole funzioni $z_{j}\left(x, g_{i}(x ; 0, \eta)\right)$, $(i, j=1, \ldots, m)$.

b) La proprietà dimostrata in a) si può in parte invertire. Si può dimostrare che, se $z_{i}(x, y),(i=1, \ldots, m)$, sono funzioni assolutamente continue rispetto alle singole variabili nel campo $D_{0}$, soddisfacenti in quasi tutto $D_{0}$ il sistema $(\mathrm{I})$ e in

$\left.{ }^{6}\right)$ Cfr. 1.c. in $\left({ }^{3}\right), \S 1$, n. 2, p. 363. Cfr. anche H. RADEMACHER, Über partielle und totale Differenzierbarkeit von Funktionen ..., Math. Ann., 79 (1919), pp. 340-359.

(7) Cir. G. Sconza Dragont, Un'osservazione sulla deriwata di una funzione composta, Rend. Sem. Mat. Univ. Padova, 20 (1951), pp. 462-467. 
$\left(-b_{0}, b_{0}\right)$ le condizioni (II), tali inoltre che, considerate le funzioni $z_{i}\left(X, g_{i}(X ; x, y)\right)$ $\left(0 \leqslant X \leqslant \beta_{i}(x, y)\right)$, esse siano assolutamente continue rispetto a $X$ in $\left(0, \beta_{i}\right)$, in tutto il campo $D_{0}$ valgono le (10) per $i=1, \ldots, m\left({ }^{3 i s}\right)$.

\section{4. - Proprietà preliminari di alcune funzioni.}

Fissato un valore intero $h$, con $1 \leqslant h \leqslant m$, e un punto $\left(x_{0}, y_{0}\right)$ in $D_{0}$, poniamo in $\left(0, a_{0}\right)$

$$
g_{h}\left(x ; x_{0}, y_{0}\right)=G(x) \text {. }
$$

Per comodità definiamo le funzioni $\phi_{j}(y),(j=1, \ldots, m)$, per qualunque $y$ ponendo

$$
\phi_{j}(y)=\phi_{j}\left(-b_{0}\right) \quad \text { per } y<-b_{0}, \quad \phi_{j}(y)=\phi_{j}\left(b_{0}\right) \quad \text { per } y>b_{0} .
$$

Vogliamo dimostrare alcune proprietà delle funzioni

$$
\phi_{j}\left(g_{i}(X ; x, y)\right) \quad(j, i=1, \ldots, m)
$$

$\mathrm{e}$

$$
\phi_{j}\left(g_{i}(X ; x, G(x))\right) \quad(j, i=1, \ldots, m) .
$$

Le funzioni $g_{i}(X ; x, y)$, come abbiamo rilevato nel $n .2$, sono monotone in $X$, e anche in $x$ e in $y$; inoltre sono equiassolutamente continue rispetto a $X$ in $\left(0, a_{0}\right)$ al variare di $(x, y)$ in $D_{\infty}$, rispetto a $x$ in $\left(0, a_{0}\right)$ al variare di $(X, y)$ in $D_{\infty}$, lipschitziane rispetto a $y$ con costante di Lipschitz indipendente da $X, \infty$.

Pertanto le funzioni (14), come funzioni assolutamente continue di una funzione equiassolutamente continua in $X$ e monotona in $X$, sono funzioni equiassolutamente continue in $X$ in $\left(0, a_{0}\right)$ al variare $d i(x, y)$ in $D_{\infty}$; analogamente risultano equiassolutamente continue rispetto a $x$ in $\left(0, a_{0}\right)$ al variare di $(X, y)$ in $D_{\infty}$, e rispetto a $y$, al variare di $X$ e $x$ in $\left(0, a_{0}\right)$.

Rileviamo inoltre che la variazione totale della funzione $\phi_{j}\left(g_{i}(X ; x, y)\right)$ coincide con la variazione totale della funzione $\phi_{i}(Y)$, per $Y$ variabile nell'intervallo $\left(g_{i}(0 ; x, y)\right.$, $\left.g_{i}\left(a_{0} ; x, y\right)\right)$.

Indicata con $V_{0 j}$ la variazione totale della funzione $\phi_{j}(Y)$ nell'intervallo $\left(-b_{0}, b_{0}\right)$ e con $V_{0}$ la massima delle $V_{0 j}(i=1,2, \ldots, m)$, per qualunque $(x, y)$ del campo $D_{0}$, indicata con $\Omega\left(\phi_{j}(\ldots)\right)$ la variazione totale rispetto a $X$ della funzione $\phi_{j}(\ldots)$, ̀̀

$$
\Omega\left(\phi_{i}\left(g_{i}(X ; x, y)\right)\right) \leqslant V_{0} \quad\left(0 \leqslant X \leqslant a_{0}\right),(i, j=1, \ldots, m) .
$$

Consideriamo ora le funzioni (15) per $i \neq h,(j=1, \ldots, m)$. La funzione $g_{i}(X ; x, G(x))$

(76is) Cfr. Ja prima memoria citata in $\left({ }^{1}\right), \S 1$, n. 26$)$. 
è monotona rispetto a $x$ in virtù dell'ipotesi (7) e di un lemma che verrà dimostrato nel $\S 2$ (cfr. n. 10) $\left(^{8}\right)$; inoltre tale funzione è assolutamente continua in $x$ : si ha infatti, per ogni coppia di valori $x^{\prime}, x^{\prime \prime}$ di $\left(0, a_{0}\right)$,

$$
\left|g_{i}\left(X ; x^{\prime \prime}, G\left(x^{\prime \prime}\right)\right)-g_{i}\left(X ; x^{\prime}, G\left(x^{\prime}\right)\right)\right| \leqslant \lambda_{1}\left|g_{i}\left(x^{\prime} ; x^{\prime \prime}, G\left(x^{\prime \prime}\right)\right)-G\left(x^{\prime}\right)\right|
$$

e

$\left|g_{i}\left(x^{\prime} ; x^{\prime \prime}, G\left(x^{\prime \prime}\right)\right)-G\left(x^{\prime}\right)\right|=\left|G\left(x^{\prime \prime}\right)+\int_{x^{\prime \prime}}^{x^{\prime}} \varrho_{i}\left(t ; g_{i}\left(t ; x^{\prime \prime}, G\left(x^{\prime \prime}\right)\right)\right) d t-\left[G\left(x^{\prime \prime}\right)+\int_{x^{\prime \prime}}^{x^{\prime}} \varrho_{n}(t, G(t)) d t\right]\right|$,

da cui, tenuto conto della (2),

$$
\left|g_{i}\left(x^{\prime} ; x^{\prime \prime}, G\left(x^{\prime \prime}\right)\right)-G\left(x^{\prime}\right)\right| \leqslant 2\left|\int_{x^{\prime}}^{x^{\prime}} M(t) d t\right|
$$

e

$$
\left|g_{i}\left(X ; x^{\prime \prime}, G\left(x^{\prime \prime}\right)\right)-g_{i}\left(X ; x^{\prime}, G\left(x^{\prime}\right)\right)\right| \leqslant 2 \lambda_{1}\left|\int_{x^{\prime}}^{x^{\prime \prime}} M(t) d t\right| .
$$

Osserviamo inoltre che le funzioni

$$
g_{i}(X ; x, G(x))=g_{i}\left(X ; x, g_{h}\left(x ; x_{0}, y_{0}\right)\right)
$$

sono equiassolutamente continue rispetto a $x$ in $\left(0, a_{0}\right)$, qualunque sia $X$ in $\left(0, a_{0}\right)$ e $\left(x_{0}, y_{0}\right)$ in $D_{\infty}$.

Tenuto conto di tali proprietà delle funzioni $g_{i}(X ; x, G(x)),(i=1, \ldots, m)$, le funzioni (15) risultano equiassolutamente continue rispetto $a$ in $\left(0, a_{0}\right)$, al variare di $X$ in $\left(0, a_{0}\right) \Theta$ di $\left(x_{0}, y_{0}\right)$ in $D_{\infty}$.

\section{5. - Esistenza della soluzione in un campo parziale.}

a) Nel presente numero restringiamo (momentaneamente) le ipotesi (6), (7): supponiamo che per quasi tutti gli $x$ di $\left(0, a_{0}\right)$, per ogni $y$ di $I_{x}$, valga una (fissata) delle (6) in corrispondenza ad ogni valore $i$, con $i=1, \ldots, m$, e che, considerata una

$\left(^{8}\right)$ Se vale la diseguaglianza $\varrho_{i}(x, y) \leqslant \varrho_{h}(x, y)$, il lemma che verrà dimostrato nel $\S 2$, n. 10, assicura che, per $x^{2}<x^{\prime \prime}$, si ha

$$
g_{i}\left(x^{\prime} ; x^{\prime \prime}, G\left(x^{\prime \prime}\right)\right) \geqslant G\left(x^{\prime}\right)
$$

e, in virtù del teorema di unicità citato in $\left({ }^{2}\right)$, è per ogni $X$ di $\left(0, a_{0}\right)$

$$
g_{i}\left(X ; x^{\prime \prime}, G\left(x^{\prime \prime}\right)\right) \geqslant g_{i}\left(X ; x^{\prime}, G\left(x^{\prime}\right)\right),
$$

vale a dire la funzione $g_{i}(X ; x, G(x))$ è non decrescente rispetto a $x$, fissato $X$.

Analogamente si prova che, se è $\varrho_{i}(x, y) \geqslant \varrho_{h}(x, y)$, la funzione $g_{i}(X ; x, G(x))$ è non crescente rispetto a $x$, fissato $X$. 
qualunque coppia di valori distinti $i, h(i, h=1, \ldots, m, i \neq h)$, per quasi tutti gli $x$ di $\left(0, a_{0}\right)$ valga, per ogni $y$ di $I_{x}$, una (fissata) delle relazioni (7).

Dimostriamo l'esistenza della soluzione, di cui al n. 1 , in un campo

$D$ :

$$
0 \leqslant x \leqslant a, \quad-b_{0}+\int_{0}^{x} M(t) d t \leqslant y \leqslant b_{0}-\int_{0}^{\infty} M(t) d t,
$$

dove $a$ è un opportuno numero positivo, non superiore ad $a_{0}$, precisato dalle (39), (44).

b) Consideriamo il sistema di equazioni integrali

$$
\begin{aligned}
& \sum_{j=1}^{m} a_{i j}(x, y) z_{j}(x, y)=\sum_{j=1}^{m} a_{i j}\left(0, g_{i}(0 ; x, y)\right) \phi_{j}\left(g_{i}(0 ; x, y)\right)+ \\
& +\int_{0}^{x} \sum_{j=1}^{m} \frac{d a_{i j}\left(X, g_{i}(X ; x, y)\right)}{d X} z_{j}\left(X, g_{i}(\ldots)\right)+ \\
& \quad+f_{i}\left(X, g_{i}(\ldots), z_{1}\left(X, g_{i}(\ldots)\right), \ldots, z_{m}(\ldots)\right) d x, \quad(i=1, \ldots, m),
\end{aligned}
$$

nelle funzioni incognite $z_{1}(x, y), \ldots, z_{m}(x, y)$.

Vogliamo costruire nel campo $D$ una soluzione del sistema (10), costituita da funzioni assolutamente continue rispetto alle singole variabili, mediante un procedimento di approssimazioni successive.

Posto in $D_{0}$

$$
z_{j}^{(0)}(x, y)=\phi_{j}(y) \quad(j=1, \ldots, m),
$$

consideriamo, per $n=1,2, \ldots$, le funzioni

$$
z_{j}^{(n)}(x, y) \quad(j=1, \ldots, m)
$$

definite in $D_{0}$ mediante le relazioni

$$
\begin{aligned}
& \sum_{j=1}^{m} a_{i j}(x, y) z_{j}^{(n)}(x, y)=\sum_{j=1}^{m} a_{i j}\left(0, g_{i}(0 ; x, y)\right) \phi_{j}\left(g_{i}(0 ; x, y)\right)+ \\
& \quad+\int_{0}^{x}\left[\sum_{j=1}^{m} \frac{d a_{i j}\left(X, g_{i}(X ; x, y)\right)}{d X} z_{j}^{(n-1)}\left(X, g_{i}(\ldots)\right)+\right. \\
& \left.\quad+f_{i}\left(X, g_{i}(\ldots), z_{1}^{(n-1)}\left(X, g_{i}(\ldots)\right), \ldots, \hat{z}_{m}^{(n-1)}\left(X, g_{i}(\ldots)\right)\right)\right] d x, \quad(i=1, \ldots, m)
\end{aligned}
$$

Vogliamo provare che le funzioni (20), definite dalle (21), costituiseono nel campo $D$, per $n=1,2, \ldots$, una successione di funzioni equilimitate, assolutamente continue rispetto a $x$ per ogni y fissato e rispetto a y per ogni $x$ fissato; che sono inoltre equiassolutamente continue in $x$ per ogni $y$ fissato $e$ in $y$ per ogni $x$ fissato, al variare $d i n$. 
Per dimostrarlo procediamo per induzione.

Nel successivo capoverso c) dimostriamo l'equilimitatezza e nel capoverso d) l'equiassoluta continuità.

c) Sia $C$ una costante positiva tale che in $\left(-b_{0}, b_{0}\right)$ sia

$$
\sum_{j=1}^{m}\left|\phi_{j}(y)\right| \leqslant C
$$

Indicati con $\alpha_{i j}(x, y)$ i complementi algebrici di $a_{i j}(x, y)$ nel determinante $A$, siano $B_{1}, B_{2}$ due costanti non negative tali che sia in tutto $D_{0}$

$$
\left|\alpha_{i j}(x, y)\right| \leqslant B_{1}, \quad\left|\alpha_{i j}(x, y)\right| \leqslant B_{2}, \quad(i, j=1, \ldots, m)
$$

Sia $\mathcal{U}_{(x)}\left(\mathrm{a}\right.$ funzione definita in $\left(0, a_{0}\right)$ dall'equazione integrale

$$
\mathcal{u}(x)=m^{2} B_{1} B_{2} C+m^{2} B_{2} \int_{0}^{x}\{[\mu(X)+A M(X)] น(X)+N(X)\} d X,
$$

vale a dire

$$
\begin{aligned}
\mathcal{U}(x)=\left\{\exp \left[m^{2} B_{2} \int_{0}^{x}(\mu(X)+\Lambda M(X)) d X\right]\right\} . \\
\cdot\left\{m^{2} B_{1} B_{2} C+m^{2} B_{2} \int_{0}^{x} N(X) \exp \left[-m^{2} B_{2} \int_{0}^{X}(\mu(t)+\Lambda M(t)) d t\right] d X\right\} .
\end{aligned}
$$

Supponiamo che le funzioni $z_{j}^{(n-1)}(x, y),(j=1, \ldots, m)$, siano continue in $D_{0}$ e soddisfino in $D_{0}$ la diseguaglianza

$$
\sup _{-b_{9}+\int_{0}^{z} M(t) d t \leqslant y \leqslant b_{0}-\int_{0}^{x} M(t) d t} \sum_{j=1}^{m}\left|z_{j}^{(n-1)}(x, y)\right| \leqslant \mathcal{U}(x) .
$$

Dimostriamo che anche le funzioni $z_{j}^{(n)}(x, y),(j=1, \ldots, m)$, soddisfano in $D_{0}$ la diseguaglianza

$$
\sum_{j=1}^{m}\left|z_{j}^{(n)}(x, y)\right| \leqslant \mathcal{U}(x)
$$

Infatti dalle (21) seguono le

$$
\begin{aligned}
& z_{j}^{(n)}(x, y)=\sum_{i=1}^{m} \alpha_{i j}(x, y)\left\{\sum_{s=1}^{m} a_{i s}\left(0, g_{i}(0 ; x, y)\right) \phi_{s}\left(g_{i}(0 ; x, y)\right)+\right. \\
& \quad+\int_{0}^{x}\left[\sum_{s=1}^{m} \frac{d a_{i s}\left(X, g_{i}(X ; x, y)\right)}{d X} z_{s}^{(n-1)}\left(X, g_{i}(\ldots)\right)+\right. \\
& \left.\left.\quad+f_{i}\left(X, g_{i}(\ldots), z_{1}^{(n-1)}(\ldots), \ldots, z_{m}^{(n-1)}(\ldots)\right)\right] d X\right\}, \quad(j=1, \ldots, m) .
\end{aligned}
$$


Dalla (25) segue

$\left|z_{j}^{(n)}(x, y)\right| \leqslant m B_{1} B_{2} C+m B_{2} \int_{0}^{n}\{(\mu(X)+\Lambda M(X)) \cup(X)+N(X)\} d X, \quad(j=1, \ldots, m)$ e anche

$$
\sum_{j=1}^{m}\left|z_{j}^{(n)}(x, y)\right| \leqslant m^{2} B_{1} B_{2} C+m^{2} B_{2} \int_{0}^{x}\{(\mu(X)+\Lambda M(X)) \cup(X)+N(X)\} d X
$$

dall'ultima diseguaglianza e dalla (24) segue la $\left(25^{\prime}\right)$.

Rileviamo che la (25) vale per le funzioni $z_{j}^{(0)}(x, y),(j=1, \ldots, m)$.

Infatti dalle $\left(1^{\prime \prime}\right)$, (23) risulta $\left(^{9}\right) m B_{1} B_{2} \geqslant 1$, e quindi dalla (24) segue $U(0) \geqslant$ $\geqslant m C>O$; tenuto conto della $(22)$ e delle $(19)$, risulta in $D_{0}$

$$
\sum_{j=1}^{m}\left|z_{j}^{(0)}(x, y)\right| \leqslant U(x)
$$

d) Indicata con $A_{0}$ la costante di Lipschitz rispetto a $y$ delle funzioni $\alpha_{i j}(x, y)$, $(i, j=1, \ldots, m)$, poniamo per brevità

$$
\begin{aligned}
& H=m \Lambda_{0}\left\{B_{1} C+\int_{0}^{a_{0}}[(\mu(X)+\Lambda M(x)) \cup(X)+N(X)] d X+m B_{2} A \mathcal{U}_{\left(a_{0}\right)+}\right. \\
& \left.+m B_{a} \lambda_{1} \int_{0}^{a_{0}} L_{1}(X) d X\right\}
\end{aligned}
$$

$$
S(t)=\left[2 \Lambda U\left(a_{0}\right)+2 \lambda_{1} \int_{0}^{a_{0}} L_{1}(\tau) d \tau\right] \cdot M(t)+[\mu(t)+\Lambda M(t)] U(t)+N(t),
$$

$$
\left(0 \leqslant t \leqslant a_{0}\right)
$$

$$
F_{1}=m B_{1} V_{0}+\int_{0}^{a_{0}} S(t) d t
$$

Sia $F_{2}$ una costante positiva maggiorante la variazione totale delle funzioni $a_{i j}\left(X, g_{n}(X ; x, y)\right),(i, j=1, \ldots, m)$, rispetto a $X$ nell'intervallo dei valori $X$, tali che il punto $\left(X, g_{k}(X ; x, y)\right)$ appartenga a $D_{0}\left({ }^{10}\right)$ : indichiamo con $\left(0, \beta_{h}(x, y)\right)$ tali intervalli.

$\left({ }^{9}\right)$ Si ha $\sum_{j=1}^{m} \alpha_{i j}(x, y) a_{i j}(x, y)=1$, e d'altra parte per le $(23)$ è $\left|\sum_{j=1}^{m} \alpha_{i j}(x, y) a_{i j}(x, y)\right| \leqslant m B_{1} B_{2}$. Quindi risulta $m B_{1} B_{2} \geqslant 1$.

(10) E immediato che la variazione totale delle funzioni $\alpha_{i j}\left(X, g_{h}(X ; x, y)\right)$ è limitata supe. riormente da una costante indipendente da $(x, y)$, perchè tale indipendenza sussiste per le funzioni $a_{r s}\left(X, g_{h}(X ; x, y)\right)$ : esse infatti sono equiassolutamente continue rispetto a $X$ al variare di $(x, y)$ in $D_{0}$. 


\section{Posto}

$$
F=V_{0}+m B_{2} F_{1}+m B_{1} F_{2} \mathrm{u}\left(a_{0}\right),
$$

sia $W$ una costante positiva, che assumiamo maggiore di $F$,

$$
W>F .
$$

Considerate le funzioni $\phi_{j}\left(g_{i}(X ; x, y)\right)$, equiassolutamente continue in $y$ (cfr. n. 4$)$, preso $\varepsilon>0$, sia $\delta$ un numero positivo che soddisfi la

$$
\delta<\frac{\varepsilon}{3\left(H+m^{2} B_{2} \Lambda \lambda_{1} W\right)},
$$

e sia inoltre tale che, se $\left(y_{1}^{\prime}, y_{1}^{\prime \prime}\right), \ldots,\left(y_{k}^{\prime}, y_{k}^{\prime \prime}\right), \ldots,\left(y_{r}^{\prime}, y_{r}^{\prime \prime}\right)$ sono $r$ intervalli non sovrapposti a due a due, con $y_{1}^{\prime}<y_{1}^{\prime \prime} \leqslant y_{2}^{\prime}<y_{2}^{n} \leqslant \ldots \leqslant y_{r}^{\prime}<y_{r}^{\prime \prime}$, e

$$
\sum_{k=1}^{r}\left|y_{k}^{\prime}-y_{k}^{\prime \prime}\right|<\delta
$$

sia

$$
B_{1} B_{2} \sum_{k=1}^{r} \sum_{i=1}^{m} \sum_{j=1}^{m}\left|\phi_{j}\left(g_{i}\left(X ; x, y_{k}^{\prime}\right)\right)-\phi_{j}\left(g_{i}\left(X ; x, y_{k}^{\prime \prime}\right)\right)\right|<\varepsilon / 3
$$

comunque siano $X, x$ in $\left(0, a_{0}\right)$.

Sia $\varepsilon_{0}$ un numero positivo fissato, e sia $\delta_{0}$ il corrispondente valore di $\delta$, determinato nel modo or ora indicato.

Sia $N_{0}$ il minimo numero intero positivo, tale che sia

$$
N_{0} \delta_{0} \geq 2 b_{0}
$$

poniamo

$$
V=N_{0} \varepsilon_{0}
$$

Oltre alle ipotesi fatte in $c$ ) sulle funzioni $z_{j}^{n-1}(x, y),(j=1, \ldots, m)$, supponiamo che tali funzioni in corrispondenza ad ogni $x$ di $(0, a)$ siano assolutamente continue rispetto a $y$ in $I_{x}$; supponiamo inoltre che le funzioni

$$
z_{j}^{(n-1)}\left(X, g_{h}(X ; x, y)\right) \quad(i, h=1, \ldots, m)
$$

siano assolutamente continue in $X\left({ }^{11}\right)$ nei rispettivi intervalli.

${ }^{(11)}$ Basterebbe che fossero a variazione limitata, agli effetti della dimostrazione.

10 - Annali di Matematica 
Supponiamo che la variazione totale delle funzioni $z_{j}^{(n-1)}(x, y),(j=1, \ldots, m)$, rispetto a $y$ nell'intervallo $I_{x}$ non superi $V$, e quella rispetto a $X$ delle funzioni (35) in $\left(0, \beta_{h}\right)$ non superi $W$ : supponiamo inoltre che, preso $\varepsilon>0$, se $\delta$ è il numero subordinato a $\varepsilon$ nel modo precedentemente indicato e se vale la (32), sia

$$
\sum_{k=1}^{r}\left|z_{j}^{(n-1)}\left(x, y_{k}^{\prime}\right)-z_{j}^{(n-1)}\left(x, y_{k}^{\prime \prime}\right)\right|<\varepsilon, \quad(j=1, \ldots, m)
$$

si intende che gli intervalli $\left(y_{1}^{\prime}, y_{1}^{\prime \prime}\right), \ldots,\left(y_{r}^{\prime}, y_{r}^{\prime \prime}\right)$ appartengono all'intervallo $I_{x}$, in corrispondenza ad ogni $x$ di $(0, a)$.

Dimostriamo

i) che è anche

$$
\sum_{k=1}^{r}\left|z_{j}^{(n)}\left(x, y_{k}^{\prime}\right)-z_{j}^{(n)}\left(x, y_{k}^{\prime \prime}\right)\right|<\varepsilon
$$

in particolare la variazione totale in $y$ delle funzioni $z_{j}^{(m)}(x, y),(j=1, \ldots, m)$, su ogni intervallo $I_{x}$ non supera $V$;

ii) che le funzioni $z_{j}^{(n)}\left(X, g_{h}(X ; x, y)\right),(j, h=1, \ldots, m)$, sono assolutamente continue in $X$ nei rispettivi intervalli $\left(0, \beta_{h}\right)$, e che la variazione totale in $X$ non supera $W$;

iii) che le funzioni $z_{j}^{(n)}(x, y),(j=1, \ldots, m)$, sono assolutamente continue in $x$, per ogni $y$ di $\left(-b_{0}, b_{0}\right)$;

iiii) che per le funzioni $z_{j}^{(0)}(x, y)=\phi_{i}(y),(j=1, \ldots, m)$, valgono le ipotesi ora formulate sulle funzioni $z_{j}^{(n-1)}(x, y),(j=1, \ldots, m)$.

i) Per ogni coppia $\left(x, y^{\prime}\right),\left(x, y^{\prime \prime}\right)$ del campo $D$ dalle $\left(21^{\prime}\right)$ si ha

$$
\begin{aligned}
& z_{j}^{(n)}\left(x, y^{\prime}\right)-z_{j}^{(n)}\left(x, y^{\prime \prime}\right)=\sum_{j=1}^{m} \alpha_{i j}\left(x, y^{\prime}\right)\left\{\sum_{s=1}^{m} a_{i s}\left(0, g_{i}\left(0 ; x, y^{\prime}\right)\right) \phi_{s}\left(g_{i}\left(0 ; x, y^{\prime}\right)\right)+\right. \\
& \quad+\int_{0}^{x}\left[\sum_{s=1}^{m} \frac{d \alpha_{i s}\left(X, g_{i}\left(X ; x, y^{\prime}\right)\right)}{d X} z_{s}^{(n-1)}\left(X, g_{i}(\ldots)\right)+\right. \\
& \left.\left.\quad+f_{i}\left(X, g_{i}(\ldots), z_{1}^{(n-1)}\left(X, g_{i}(\ldots)\right), \ldots, z_{m}^{(n-1)}(\ldots)\right)\right] d X\right\}- \\
& \quad-\sum_{i=1}^{m} \alpha_{i j}\left(x, y^{\prime \prime}\right)\left\{\sum_{s=1}^{m} a_{i s}\left(0, g_{i}\left(0 ; x, y^{\prime \prime}\right)\right) \phi_{s}\left(g_{i}\left(0 ; x, y^{\prime \prime}\right)\right)+\right. \\
& \left.\quad+\int_{0}^{x}\left[\sum_{s=1}^{m} \frac{d a_{i s}\left(X, g_{i}\left(X ; x, y^{\prime \prime}\right)\right)}{d X} z_{s}^{(n-1)}\left(X, g_{i}(\ldots)\right)+f_{i}\left(X, g_{i}(\ldots), z_{1}^{(n-1)}(\ldots), z_{m}^{(n-1)}(\ldots)\right)\right] d X\right\}= \\
& \quad=\sum_{i=1}^{m}\left[\alpha_{i j}\left(x, y^{\prime}\right)-\alpha_{i j}\left(x, y^{\prime \prime}\right)\right]\left\{\sum_{s=1}^{m} a_{i s}\left(0, g_{i}\left(0 ; x, y^{\prime}\right)\right) \phi_{s}\left(g_{i}\left(0 ; x, y^{\prime}\right)\right)+\right.
\end{aligned}
$$




$$
\begin{aligned}
& +\int_{0}^{n}\left[\sum_{s=1}^{m} \frac{d a_{i s}\left(X, g_{i}\left(X ; x, y^{\prime}\right)\right)}{d X} z_{s}^{(n-1)}\left(X, g_{i}(\ldots)\right)+\right. \\
& \left.\left.+f_{i}\left(X, g_{i}(\ldots), z_{1}^{(n-1)}(\ldots), \ldots, z_{m}^{(n-1)}(\ldots)\right)\right] d X\right\}+ \\
& +\sum_{i=1}^{m} \alpha_{i j}\left(x, y^{\prime \prime}\right)\left\{\sum_{s=1}^{m} a_{i s}\left(0, g_{i}\left(0 ; x, y^{\prime}\right)\right) \phi_{s}\left(g_{i}\left(0 ; x, y^{\prime}\right)\right)-\right. \\
& -\sum_{s=1}^{m} a_{i s}\left(0, g_{i}\left(0 ; x, y^{\prime \prime}\right)\right) \phi_{s}\left(g_{i}\left(0 ; x, y^{\prime \prime}\right)\right)+ \\
& +\int_{0}^{x}\left[\sum_{s=1}^{m} \frac{d a_{i s}\left(X, g_{i}\left(X ; x, y^{\prime}\right)\right)}{d X} z_{s}^{(n-1)}\left(X, g_{i}\left(X ; x, y^{\prime}\right)\right)+\right. \\
& +f_{i}\left(X, g_{i}\left(X ; x, y^{\prime}\right), z_{1}^{(n-1)}(\ldots), \ldots, z_{m}^{(n-1)}(\ldots)\right)- \\
& -\sum_{s=1}^{m_{n}} \frac{d a_{i s}\left(X, g_{i}\left(X ; x, y^{\prime \prime}\right)\right)}{d X} z_{s}^{(n-1)}\left(X, g_{i}\left(X ; x, y^{\prime \prime}\right)\right)- \\
& \left.\left.\left.\quad-f_{i}\left(X, g_{i}\left(X ; x, y^{\prime \prime}\right)\right), z_{1}^{(n-1)}(\ldots), \ldots, z_{m}^{(n-1)}(\ldots)\right)\right] d X\right\}
\end{aligned}
$$

Aggiungendo e togliendo termini opportuni, operando un'integrazione per parti, parti, tenendo conto delle (22), (23), (1), (2), (4), (5) ed essendo per ipotesi $\left({ }^{11 b i s}\right)$

$$
\int_{0}^{x}\left|\frac{d z_{s}^{(n-1)}\left(X, g_{i}(X ; x, y)\right)}{d X}\right| d X \leqslant W,
$$

si ottiene

$$
\begin{aligned}
& \left|z_{j}^{(n)}\left(x, y^{\prime}\right)\right|-z_{j}^{(n)}\left(x, y^{\prime \prime}\right)\left|\leqslant m \Lambda_{0}\right| y^{\prime}-y^{\prime \prime} \mid\left\{B_{1} O+\int_{0}^{a_{0}}[(\mu(X)+\Lambda M(X)) \mathcal{U}(X)+N(X)] d X\right\}+ \\
& +\mid \sum_{i=1}^{m} \alpha_{i j}\left(x, y^{\prime \prime}\right)\left\{\sum_{s=1}^{m}\left[a_{i s}\left(0, g_{i}\left(0 ; x, y^{\prime}\right)\right) \phi_{s}\left(g i\left(0 ; x, y^{\prime}\right)\right)-a_{i s}\left(0, g_{i}\left(0 ; x, y^{\prime \prime}\right)\right) \phi_{s}\left(g_{i}\left(0 ; x, y^{\prime \prime}\right)\right)\right]+\right. \\
& +\sum_{s=1}^{m}\left[\left(a_{i s}\left(X, g_{i}\left(X ; x, y^{\prime}\right)\right)-a_{i s}\left(X, g_{i}\left(X ; x, y^{\prime \prime}\right)\right)\right) z_{8}^{(n-1)}\left(X, g_{i}\left(X ; x, y^{\prime \prime}\right)\right)\right]_{X=0}^{X=x}- \\
& \left.-\int_{0}^{\infty} \sum_{s=1}^{m}\left(a_{i s}\left(X, g_{i}\left(X ; x, y^{\prime}\right)\right)-a_{i s}\left(X, g_{i}\left(X ; x, y^{\prime \prime}\right)\right)\right) \frac{d z_{s}^{(n-1)}\left(X, g_{i}\left(X ; x, y^{\prime \prime}\right)\right)}{d X} d X\right\} \mid+
\end{aligned}
$$

${ }^{(11 b i s)}$ La variazione della funzione $z_{s}^{(n-1)}\left(X, g_{i}(X ; x, y)\right)$ rispetto a $X$ in $(0, x)$ è uguale a

$$
\int_{0}^{x}\left|\frac{d z_{s}^{(n-1)}\left(X, g_{i}(X ; x, y)\right)}{d X}\right| d X .
$$


$+B_{2} \sum_{i=1}^{m} \int_{0}^{k} L_{1}(X)\left|g_{i}\left(X ; x, y^{\prime}\right)-g_{i}\left(X ; x, y^{\prime \prime}\right)\right| d X+$
$+B_{2} \sum_{i=1}^{m} \int_{0}^{x}\left(\mu(X)+A M(X)+L_{1}(X)\right) \sum_{s=1}^{m} \mid \xi_{s}^{(n-1)}\left(X, g_{i}\left(X ; x, y^{\prime}\right)\right)-$ $-z_{s}^{(n-1)}\left(X, g_{i}\left(X ; x, y^{\prime \prime}\right)\right) \mid d x \leqslant$

$\leqslant m A_{0}\left\{B_{1} C+\int_{0}^{a_{0}}[(\mu(X)+\Lambda M(X)) \varkappa(X)+N(X)] d X\right\} \cdot\left|y^{\prime}-y^{\prime \prime}\right|+$

$+B_{1} B_{2} \sum_{i=1}^{m} \sum_{s=1}^{m}\left|\phi_{s}\left(g_{i}\left(0 ; x, y^{\prime}\right)\right)-\phi_{s}\left(g_{i}\left(0 ; x, y^{\prime \prime}\right)\right)\right|+$

$+m B_{2} \Lambda U\left(a_{0}\right)\left|y^{\prime}-y^{\prime \prime}\right|+m^{2} B_{2} \Lambda \lambda_{1} W\left|y^{\prime}-y^{\prime \prime}\right|+m B_{2} \lambda_{1} \int_{0}^{a_{8}} L_{1}(X) d X \cdot\left|y^{\prime}-y^{\prime \prime}\right|+$

$+B_{2} \sum_{i=1}^{m} \int_{0}^{x}\left(\mu(X)+\Lambda M(X)+L_{1}(x)\right) \sum_{s=1}^{m}\left|z_{s}^{(n-1)}\left(X, g_{i}\left(X ; x, y^{\prime}\right)\right)-z_{s}^{(n-1)}\left(X, g_{i}\left(X ; x, y^{\prime \prime}\right)\right)\right| d X$

Avendo posto (cfr. la (26))

$H=m \Lambda_{0}\left\{B_{1} C+\int_{0}^{a_{a}}[(\mu(X)+\Lambda M(X)) \cup(X)+N(X)] d X\right\}+m B_{2} A \cup\left(a_{0}\right)+m B_{2} \lambda_{1} \int_{0}^{a_{0}} L_{1}(X) d X$, si ottiene

$$
\begin{aligned}
& \left|z_{j}^{(n)}\left(x, y^{\prime}\right)-z_{j}^{(n)}\left(x, y^{\prime \prime}\right)\right| \leqslant H\left|y^{\prime}-y^{\prime \prime}\right|+m^{2} B_{2} \Lambda \lambda_{1} W\left|y^{\prime}-y^{\prime \prime}\right|+ \\
& +B_{1} B_{2} \sum_{i=1}^{m} \sum_{s=1}^{m}\left|\phi_{s}\left(g_{i}\left(0 ; x, y^{\prime}\right)\right)-\phi_{s}\left(g_{i}\left(0 ; x, y^{\prime \prime}\right)\right)\right|+ \\
& +B_{2} \sum_{i=1}^{m} \int_{0}^{x}\left(\mu(X)+\Lambda M(X)+L_{1}(X)\right) \sum_{s=1}^{m}\left|z_{s}^{(n-1)}\left(X, g_{i}\left(X ; x, y^{\prime}\right)\right)-z_{s}^{(n-1)}\left(X, g_{i}\left(X ; x, y^{\prime \prime}\right)\right)\right| d X,
\end{aligned}
$$

Si ha quindi

$$
\begin{aligned}
\sum_{k=1}^{r} \mid z_{j}^{(n)}\left(x, y_{k}^{\prime}\right) & -z_{j}^{(n)}\left(x, y_{k}^{\prime \prime}\right)\left|\leqslant\left(H+m^{2} B_{2} \Lambda \lambda_{1} W\right) \sum_{k=1}^{r}\right| y_{k}^{\prime}-y_{k}^{\prime \prime} \mid+ \\
& +B_{1} B_{2} \sum_{k=1}^{r} \sum_{i=1}^{m} \sum_{s=1}^{m}\left|\phi_{s}\left(g_{i}\left(0 ; x, y_{k}^{\prime}\right)\right)-\phi_{s}\left(g_{i}\left(0 ; x, y_{k}^{\prime \prime}\right)\right)\right|+ \\
& +B_{2} \sum_{k=1}^{r} \int_{0}^{x}\left(\mu(X)+\Lambda M(X)+L_{1}(X)\right) \sum_{i=1}^{m} \sum_{s=1}^{m} \mid z_{s}^{(n-1)}\left(X, g_{i}\left(X ; x, y_{k}^{\prime}\right)\right)- \\
& \quad-z_{s}^{(n-1)}\left(X, g_{i}\left(X ; x, y_{k}^{\prime \prime}\right)\right) \mid d X, \quad(j=1, \ldots, m)
\end{aligned}
$$


Osserviamo che, tenuto conto delle (9), (32), è

$$
\sum_{k=1}^{r}\left|g_{i}\left(X ; x, y_{k}^{\prime}\right)-g_{i}\left(X ; x, y_{k}^{\prime \prime}\right)\right| \leqslant \lambda_{1} \sum_{k=1}^{r}\left|y_{k}^{\prime}-y_{k}^{\prime \prime}\right|<\lambda_{1} \delta, \quad(0 \leqslant X \leqslant x),
$$

e osserviamo anche che in virtù del citato teorema di unicità relativo all'equazione integrale (8) è

$$
\begin{aligned}
g_{i}\left(X ; x, y_{k}^{\prime}\right)<g_{i}\left(X ; x, y_{k}^{\prime \prime}\right) \leqslant g_{i}\left(X ; x, y_{k+1}^{\prime}\right)<g_{i}\left(X ; x, y_{k+1}^{\prime \prime}\right), \quad(0 \leqslant X \leqslant x) \\
(k=1,2, \ldots, r-1, i=1, \ldots, m),
\end{aligned}
$$

cioè gli intervalli $\left(g_{i}\left(X ; x, y_{k}^{\prime}\right), g_{i}\left(X ; x, y_{k}^{\prime \prime}\right)\right),(k=1, \ldots, r)$, non si sovrappongono e hanno ampiezza complessiva minore di $\lambda_{1} \delta$.

Indicato con $N_{\lambda_{1}}$ il minimo intero positivo non inferiore a $\lambda_{1}$, tenuto conto delle (36) ̀̀

$$
\sum_{k=1}^{r}\left|z_{j}^{(n-1)}\left(X, g_{i}\left(X ; x, y_{k}^{\prime}\right)\right)-z_{j}^{(n-1)}\left(X, g_{i}\left(X ; x, y_{k}^{\prime \prime}\right)\right)\right|<N_{\lambda_{1}} \varepsilon, \quad(0 \leqslant X \leqslant x),
$$

come si vede intercalando eventualmente ai punti $\left(g_{i}\left(X ; x, y_{k}^{\prime}\right), g_{i}\left(X ; x, y_{k}^{\prime \prime}\right)\right)$, sulla retta dei punti di ascissa $X$, altri punti in modo opportuno, in modo cioè da decomporre l'insieme di intervalli $\left(g_{i}\left(X ; x, y_{k}^{\prime}\right), g_{i}\left(X ; x, y_{k}^{\prime \prime}\right)\right),(k=1, \ldots, r)$, in $N_{\lambda_{1}}$ insiemi di intervalli non sovrapponentisi

$$
\left(\eta_{t 1}^{\prime}, \eta_{t 1}^{\prime \prime}\right), \quad\left(\eta_{t 2}^{\prime}, \eta_{t 2}^{\prime \prime}\right), \ldots,\left(\eta_{t v_{t}}^{\prime}, \eta_{t v_{\ell}}^{\prime \prime}\right), \quad\left(t=1, \ldots, N_{\lambda_{1}}\right),
$$

ciascun insieme dei quali abbia ampiezza minore di $\delta$, per cui risulta

$$
\sum_{h=1}^{v_{t}}\left|z_{j}^{(n-1)}\left(X, \eta_{i \hbar}^{\prime}\right)-z_{j}^{(n-1)}\left(X, \eta_{i h}^{\prime \prime}\right)\right|<\varepsilon, \quad\left(t=1, \ldots, N_{\lambda_{1}}\right)
$$

e anche

$$
\begin{aligned}
\sum_{k=1}^{*}\left|z_{j}^{(n-1)}\left(X, g_{i}\left(X ; x, y_{k}^{\prime}\right)\right)-z_{j}^{(n-1)}\left(X, g_{i}\left(X ; x, y_{k}^{\prime \prime}\right)\right)\right| \leqslant & \\
& \leqslant \sum_{i=1}^{N_{k_{1}}} \sum_{h=1}^{\eta_{t}}\left|z_{j}^{(n-1)}\left(X, \eta_{t h}^{i}\right)-z_{j}^{(n-1)}\left(X, \eta_{t h}^{\prime \prime}\right)\right|<N_{i_{1}} \cdot \varepsilon .
\end{aligned}
$$

Tenuto conto delle (31), (32), (33), (37) si ha allora

$$
\begin{array}{r}
\sum_{k=1}^{r}\left|z_{j}^{(n)}\left(x, y_{k}^{\prime}\right)-z_{j}^{(n)}\left(x, y_{k}^{\prime \prime}\right)\right|<\frac{2}{3} \varepsilon+m^{2} B_{2} N_{\lambda_{1}} \varepsilon \int_{0}^{x}\left(\mu(X)+\Lambda M(X)+L_{1}(X)\right) d X, \\
(j=1, \ldots, m),
\end{array}
$$

e, se $a$ è il massimo numero positivo, non superiore ad $a_{0}$, tale che sia

$$
m^{2} B_{2} N_{\lambda_{1}} \int_{0}^{a}\left(\mu(x)+A M(X)+L_{1}(x)\right) d X \leqslant \frac{1}{3}
$$


risulta

$$
\sum_{k=1}^{r}\left|z_{j}^{(n)}\left(x, y_{k}^{\prime}\right)-z_{j}^{(n)}\left(x, y_{k}^{\prime \prime}\right)\right|<\varepsilon, \quad(j=1, \ldots, m)
$$

come si voleva dimostrare.

Nel caso particolare in cui per $\varepsilon$ si prenda il valore $\varepsilon_{0}$, dove $\varepsilon_{0}$ è il numero positivo fissato una volta per tutte all'inizio del presente capoverso $d$ ), e sia

$$
\sum_{k=1}^{r}\left|y_{k}^{\prime}-y_{k}^{\prime \prime}\right|<\delta_{0}
$$

risulta

$$
\sum_{k=1}^{r}\left|z_{j}^{(n)}\left(x, y_{k}^{l}\right)-z_{j}^{(n)}\left(x, y_{k}^{\prime \prime}\right)\right|<\varepsilon_{0}, \quad(j=1, \ldots, m) ;
$$

pertanto la variazione totale delle funzioni $z_{j}^{(n)}(x, y)$ rispetto a $y$, nell'intervallo $I_{x}$, non supera $N_{0} \varepsilon_{0}$, cioè $V$.

ii) Dimostriamo ora che le funzioni $z_{j}^{(n)}\left(X, g_{i}(X ; x, y)\right),(i, j=1, \ldots, m)$, sono assolutamente continue in $X$ nei rispettivi intervalli $\left(0, \beta_{i}\right)$ e che la loro variazione totale rispetto a $X$ non supera $W$.

Considerata la curva caratteristica

$$
Y=g_{h}(X ; x, y) \quad\left(0 \leqslant X \leqslant \beta_{h}\right),
$$

siano $X^{\prime}, X^{\prime \prime}$, con $X^{\prime}<X^{\prime \prime}$, due valori di $\left(0, \beta_{n}\right)$.

Posto, in modo analogo al n. 4 ,

$$
\begin{aligned}
& g_{h}(X ; x, y)=G(X) \quad\left(0 \leqslant X \leqslant \beta_{h}\right), \\
& G\left(X^{\prime}\right)=Y^{\prime}, \quad G\left(X^{\prime \prime}\right)=Y^{\prime \prime},
\end{aligned}
$$

dalla $i$-ma delle (21), nella quale si pongano $X, G(X)$ al posto di $x, y$ e si indichi con $t$ la variabile di integrazione, si ha $\left({ }^{12}\right)$, per $i \neq h$,

$$
\begin{aligned}
& \sum_{j=1}^{m} a_{i j}\left(X^{\prime}, G\left(X^{\prime}\right)\right) z_{j}^{(n)}\left(X^{\prime}, G\left(X^{\prime}\right)\right)-\sum_{j=1}^{m} a_{i j}\left(X^{\prime \prime}, G\left(X^{\prime \prime}\right)\right) z_{j}^{(n)}\left(X^{\prime \prime}, G\left(X^{\prime \prime}\right)\right)= \\
& \quad=\sum_{j=1}^{m} a_{i j}\left(0, g_{i}\left(0 ; X^{\prime}, Y^{\prime}\right)\right) \phi_{j}\left(g_{i}\left(0 ; X^{\prime}, Y^{\prime}\right)\right)+
\end{aligned}
$$

(12) Come abbiamo già osservato (cfr. n. 2) il campo $D$ è tale che per ogni $(x . y)$ di $D$, per ogni $X$ con $0 \leqslant X \leqslant x$, i punti delle curve

$$
Y=g_{i}(X ; x, y) \quad(i=1, \ldots, m)
$$

appartengono al campo stesso.

In particolare allora, essendo $X^{\prime}<X^{\prime \prime}$, il punto

appartiene al campo $D$.

$$
\left(X^{\prime}, g_{i}\left(X^{\prime} ; X^{\prime \prime}, Y^{\prime \prime}\right)\right)
$$




$$
\begin{aligned}
& +\int_{0}^{X^{\prime}}\left\{\sum_{j=1}^{m} \frac{d a_{i j}\left(t, g_{i}\left(t ; X^{\prime}, Y^{\prime}\right)\right)}{d t} z_{j}^{(n-1)}\left(t, g_{i}(\ldots)\right)+f_{i}\left(t, g_{i}(\ldots), z_{1}^{(n-1)}(\ldots), \ldots, z_{m}^{(n-1)}(\ldots)\right)\right\} d t- \\
& -\sum_{j=1}^{m}\left[a_{i j}\left(0, g_{i}\left(0 ; X^{\prime \prime}, Y^{\prime \prime}\right)\right) \phi_{j}\left(g_{i}\left(0 ; X^{\prime \prime}, T^{\prime \prime}\right)\right)-\int_{0}^{X^{\prime \prime}} \int_{j=1}^{m} \frac{d a_{i j}\left(t, g_{i}\left(t ; X^{\prime \prime}, Y^{\prime \prime}\right)\right)}{d t}\right. \\
& \left.\left.\cdot z_{j}^{(n-1)}\left(t, g_{i}(\ldots)\right)+f_{i}\left(t, g_{i}(\ldots)\right), z_{i}^{(n-1)}(\ldots), \ldots, z_{m}^{(n-1)}(\ldots)\right)\right\} d t= \\
& =\sum_{j=1}^{m}\left[a_{i j}\left(0, g_{i}\left(0 ; X^{\prime}, Y^{\prime}\right)\right) \phi_{i}\left(g_{i}\left(0 ; X^{\prime}, Y^{\prime}\right)\right)-a_{i j}\left(0, g_{i}\left(0 ; X^{\prime \prime}, Y^{\prime \prime}\right)\right) \phi_{j}\left(g_{i}\left(0 ; X^{\prime \prime}, Y^{\prime \prime}\right)\right)\right]+ \\
& +\sum_{j=1}^{n}\left[\left(a_{i j}\left(t, g_{i}\left(t ; X^{\prime}, Y^{\prime}\right)\right)-a_{i j}\left(t, g_{i}\left(t ; X^{n}, Y^{n}\right)\right)\right) z_{j}^{(n-1)}\left(t, g_{i}\left(t ; X^{\prime}, Y^{\prime}\right)\right)\right]_{t=0}^{t=X^{\prime}}- \\
& -\int_{0}^{X^{\prime}} \sum_{j=1}^{m}\left[a_{i s}\left(t, g_{i}\left(t ; X^{\prime}, Y^{\prime}\right)\right)-a_{i j}\left(t, g_{i}\left(t ; X^{\prime \prime}, Y^{\prime \prime}\right)\right)\right] \cdot \frac{d^{(n-1)}\left(t, g_{i}\left(t ; X^{\prime}, Y^{\prime}\right)\right)}{d t} d t+ \\
& +\int_{0}^{X^{*}}\left\{\sum_{j=1}^{m b} \frac{d a_{i j}\left(t, g_{i}\left(t ; X^{\prime \prime}, Y^{\prime \prime}\right)\right)}{d t}\left[z_{j}^{(n-1)}\left(t, g_{i}\left(t ; X^{\prime}, Y^{\prime}\right)\right)-z_{j}^{(n-1)}\left(t, g_{i}\left(t ; X^{\prime \prime}, Y^{\prime \prime}\right)\right)\right]+\right. \\
& +f_{i}\left(t, g_{i}\left(t ; X^{\prime}, Y^{\prime}\right), z_{1}^{(n-1)}(\ldots), \ldots, z_{m}^{(n-1)}(\ldots)\right)- \\
& \left.-f_{i}\left(t, g_{i}\left(t ; X^{\prime \prime}, X^{\prime \prime}\right), z_{1}^{(n-1)}(\ldots), \ldots, z_{m}^{(n-1)}(\ldots)\right)\right\} d t+ \\
& +\int_{X^{\prime}}^{X^{*}}\left\{\sum_{j=1}^{n} \frac{d a_{i j}\left(t, g_{i}\left(t ; X^{\prime \prime}, X^{\prime \prime}\right)\right)}{d t} z_{j}^{(n-1)}\left(t, g_{i}(\ldots)\right)+f_{i}\left(t, g_{i}(\ldots), z_{1}^{(n-1)}(\ldots), \ldots, z_{m}^{(n-1)}(\ldots)\right) d t\right. \text {. }
\end{aligned}
$$

Tenendo conto delle $(22),(23),(1),(2),(4),(5),(17),(18)$ semplificando si ottiene

$$
\begin{aligned}
& \left|\sum_{j=1}^{m} a_{z j}\left(X^{\prime}, G\left(X^{\prime}\right)\right) \approx_{j}^{(n)}\left(X^{\prime}, G\left(X^{\prime}\right)\right)-\sum_{j=1}^{m} a_{i j}\left(X^{\prime \prime}, G\left(X^{\prime \prime}\right)\right) z_{j}^{(n)}\left(X^{\prime \prime}, G\left(X^{\prime \prime}\right)\right)\right| \leqslant \\
& \leqslant B_{1} \sum_{j=1}^{m}\left|\phi_{j}\left(g_{i}\left(0 ; X^{\prime}, Y^{\prime}\right)\right)-\phi_{i}\left(g_{i}\left(0 ; X^{\prime \prime}, Y^{\prime \prime}\right)\right)\right|+\Lambda U\left(X^{\prime}\right)\left|g_{i}\left(X^{\prime} ; X^{\prime \prime}, Y^{\prime \prime}\right)-Y^{\prime}\right|+ \\
& +\int_{0}^{X^{\prime}}\left\{A\left|g_{i}\left(t ; X^{\prime}, Y^{\prime}\right)-g_{i}\left(t ; X^{\prime \prime}, Y^{\prime \prime}\right)\right| \sum_{j=1}^{m} \frac{d z_{j}^{(n-1)}\left(t, g_{i}\left(t ; X^{\prime}, Y^{\prime}\right)\right)}{d t}+\right. \\
& +\left[\mu(t)+\Lambda M(t)+L_{1}(t)\right] \sum_{j=1}^{m}\left|z_{j}^{(n-1)}\left(t, g_{i}\left(t ; X^{\prime}, Y^{\prime}\right)\right)-z_{j}^{(n-1)}\left(t, g_{i}\left(t ; X^{\prime \prime}, Y^{\prime \prime}\right)\right)\right|+ \\
& \left.\left.+L_{1}(t) \mid g_{i}\left(t ; X^{\prime}, Y^{\prime}\right)-g_{i}\left(t ; X^{\prime \prime}, Y^{\prime \prime}\right)\right\}\right\} d t+\int_{X^{\prime}}^{X^{\prime \prime}}[(\mu(t)+\Lambda M(t)) \cup(t)+N(t)] d t \leqslant \\
& \leqslant B_{1} \sum_{j=1}^{m}\left|\phi_{j}\left(g_{i}\left(0 ; X^{\prime}, Y^{\prime}\right)\right)-\phi_{j}\left(g_{i}\left(0 ; X^{\prime \prime}, X^{\prime \prime}\right)\right)\right|+
\end{aligned}
$$




$$
\begin{array}{r}
+\int_{X^{\prime}}^{X^{\prime \prime}}\left\{\left[2 \Lambda U\left(a_{0}\right)+2 \lambda_{1} \int_{0}^{a_{0}} L_{1}(\tau) d \tau\right] M(t)+[\mu(t)+\Lambda M(t)] U(t)+N(t)\right\} d t+ \\
+2 m \Lambda \lambda_{1} W \int_{X^{\prime}}^{X^{\prime \prime}} M(t) d t+ \\
+\int_{0}^{X^{\prime}}\left[\mu(t)+\Lambda M(t)+L_{1}(t)\right] \sum_{j=1}^{m}\left|z_{j}^{(n-1)}\left(t, g_{i}\left(t ; X^{\prime}, Y^{\prime}\right)\right)-z_{j}^{(n-1)}\left(t, g_{i}\left(t ; X^{\prime \prime}, Y^{\prime \prime}\right)\right)\right| d t .
\end{array}
$$

Avendo posto

$$
S(t)=\left[2 \Lambda U\left(a_{0}\right)+2 \lambda_{1} \int_{0}^{a_{0}} L_{1}(\tau) d \tau\right] M(t)+[\mu(t)+\Lambda M(t)] น(t)+N(t),
$$

risulta

$$
\begin{aligned}
& \left|\sum_{j=1}^{m} a_{i j}\left(X^{\prime}, G\left(X^{\prime}\right)\right) z_{j}^{(n)}\left(X^{\prime}, G\left(X^{\prime}\right)\right)-\sum_{j=1}^{m} a_{i j}\left(X^{\prime \prime}, G\left(X^{\prime \prime}\right)\right) z_{j}^{(n)}\left(X^{\prime \prime}, G\left(X^{\prime \prime}\right)\right)\right| \leqslant \\
& \quad \leqslant \int_{X^{\prime}}^{X^{\prime \prime}} S(t) d t+2 m \Lambda \lambda_{1} W \int_{X^{\prime}}^{X^{\prime}} M(t) d t+B_{1} \sum_{j=1}^{m}\left|\phi_{j}\left(g_{i}\left(0 ; X^{\prime}, Y^{\prime}\right)\right)-\phi_{j}\left(g_{i}\left(0 ; X^{\prime \prime}, Y^{\prime \prime}\right)\right)\right|+ \\
& \quad+\int_{0}^{X^{\prime}}\left[\mu(t)+\Lambda M(t)+L_{1}(t)\right] \sum_{j=1}^{m}\left|z_{j}^{(n-1)}\left(t, g_{i}\left(t ; X^{\prime}, X^{\prime}\right)\right)-z_{j}^{(n-1)}\left(t, g_{i}\left(t ; X^{\prime \prime}, X^{\prime \prime}\right)\right)\right| d t .
\end{aligned}
$$

Consideriamo ora un insieme di intervalli di $\left(0, \beta_{h}\right)$

$$
\left(X_{1}^{\prime}, X_{1}^{\prime \prime}\right), \quad\left(X_{2}^{\prime}, X_{2}^{\prime \prime}\right), \ldots,\left(X_{r}^{\prime}, X_{r}^{\prime \prime}\right)
$$

non sovrapposti a due a due, con $X_{1}^{\prime}<X_{1}^{\prime \prime} \leqslant X_{2}^{\prime}<X_{2}^{\prime \prime} \leqslant \ldots \leqslant X_{r}^{\prime}<X_{r}^{\prime \prime}$.

Posto

$$
g_{h}\left(X_{k}^{\prime} ; x, y\right)=Y_{k}^{\prime \prime}, \quad g_{h}\left(X_{k}^{\prime} ; x, y\right)=Y_{k}^{\prime \prime}, \quad(k=1, \ldots, r),
$$

dalla (40) si ha

$$
\begin{aligned}
& \sum_{k=1}^{r}\left|\sum_{j=1}^{m} a_{i j}\left(X_{k}^{\prime}, Y_{k}^{\prime}\right) z_{j}^{(n)}\left(X_{k}^{\prime}, Y_{k}^{\prime}\right)-\sum_{j=1}^{m} a_{i j}\left(X_{k}^{\prime \prime}, Y_{k}^{\prime \prime}\right) z_{j}^{(n)}\left(X_{k}^{\prime \prime}, Y_{k}^{\prime \prime}\right)\right| \leqslant \\
\leqslant & \sum_{k=1}^{r} \int_{X_{k}^{\prime}}^{x_{k}^{\prime \prime}} S(t) d t+2 m \Lambda \lambda_{1} W \sum_{k=1}^{r} \int_{X_{k}^{\prime}}^{x_{k}^{\prime \prime}} M(t) d t+ \\
+ & B_{1} \sum_{k=1}^{r} \sum_{j=1}^{m}\left|\phi_{i}\left(g_{i}\left(0 ; X_{k}^{\prime}, Y_{k}^{\prime}\right)\right)-\phi_{j}\left(g_{i}\left(0 ; X_{k}^{\prime \prime}, Y_{k}^{\prime \prime}\right)\right)\right|+ \\
+ & \sum_{k=1}^{r} \int_{0}^{x_{k}^{\prime}}\left[\mu(t)+\Lambda M(t)+L_{1}(t)\right] \sum_{j=1}^{m}\left|z_{j}^{(n-1)}\left(t, g_{i}\left(t ; X_{k}^{\prime}, Y_{k}^{\prime}\right)\right)-z_{j}^{(n-1)}\left(t, g_{i}\left(t ; X_{k}^{\prime \prime}, Y_{k}^{\prime \prime}\right)\right)\right| d t .
\end{aligned}
$$


Osserviamo che, in virtù dell'ipotesi (7), tenuto conto del lemma del $\S 2$, n. 3 (cfr. anche nota $\left.{ }^{8}\right)$ ), per qualunque coppia di valori $X^{\prime}, X^{\prime \prime}$ di $\left(0, a_{0}\right)$, con $X^{\prime}<X^{\prime \prime}$, ̀̀

$$
g_{i}\left(X^{\prime} ; X^{\prime \prime}, Y^{\prime \prime}\right) \geqslant X^{\prime}
$$

ovvero

$$
g_{i}\left(X^{\prime} ; X^{\prime \prime}, X^{\prime \prime}\right) \leqslant \bar{X}^{\prime}
$$

(a seconda che valga la $\varrho_{i}(x, y) \leqslant \varrho_{n}(x, y)$ o la $\left.\varrho_{i}(x, y) \geqslant \varrho_{n}(x, y)\right)$.

Allora, essendo $g_{i}\left(X^{\prime} ; X^{\prime}, \bar{Y}^{\prime}\right)=\bar{Y}^{\prime}$ in virtù del teorema di unicità citato in $\left(^{2 b i s}\right)$, ̀̀

$$
g_{i}\left(t ; X^{\prime \prime}, Y^{\prime \prime}\right) \geqslant g_{i}\left(t ; X^{\prime}, Y^{\prime}\right)
$$

ovvero

$$
g_{i}\left(t ; X^{\prime \prime}, Y^{\prime \prime}\right) \leqslant g_{i}\left(t ; X^{\prime}, Y^{\prime}\right)
$$

per ogni $t$ di $\left(0, a_{0}\right)$.

Pertanto si ha in $\left(0, a_{0}\right)$

$$
g_{i}\left(t ; X_{1}^{\prime}, Y_{1}^{\prime}\right) \leqslant g_{i}\left(t ; X_{1}^{\prime \prime}, Y_{1}^{\prime \prime}\right) \leqslant g_{i}\left(t ; X_{2}^{\prime}, Y_{2}^{\prime}\right) \leqslant \ldots \leqslant g_{i}\left(t ; X_{r}^{\prime \prime}, Y_{r}^{\prime \prime}\right)
$$

ovvero

$$
g_{i}\left(t ; X_{1}^{\prime}, Y_{1}^{\prime}\right) \geqslant g_{i}\left(t ; X_{1}^{\prime \prime}, Y_{1}^{\prime \prime}\right) \geqslant g_{i}\left(t ; X_{2}^{\prime}, Y_{2}^{\prime}\right) \geqslant \ldots \geqslant g_{i}\left(t ; X_{r}^{\prime \prime}, Y_{r}^{\prime \prime}\right),
$$

vale a dire le caratteristiche $Y=g_{i}\left(t ; X_{k}^{\prime}, Y_{k i}^{\prime}\right), Y=g_{i}\left(t ; X_{k}^{\prime \prime}, Y_{k}^{\prime \prime}\right),(k=1, \ldots, r)$, intercettano sulle rette $x=t$, per ogni $t$ di $\left(0, a_{0}\right), r$ segmenti (eventualmente alcuni di ampiezza nulla) non sovrapposti a due a due.

Ciascuna delle funzioni

$$
z_{j}^{(n-1)}\left(t, g_{i}(t ; X, G(X))\right), \quad(j=1, \ldots, m),
$$

fissato $t$, è assolutamente continua rispetto a $X$ in $\left(0, \beta_{k}\right)$, come funzione assolutamente continua (per l'ipotesi fatta sulle funzioni $z_{j}^{(n-1)}(t, Y)$ ) di una funzione

$$
g_{i}(t ; X, G(X))
$$

monotona in $X$ e assolutamente continua in $X$ (cfr. n. 4); inoltre è equiassolutamente continua in $X$, per qualunque $t$ di $\left(0, \beta_{h}\right)$, perchè sia la funzione $g_{i}(t ; X, G(X))$ che la funzione $z_{j}^{n-1}(t, Y)$ sono equiassolutamente continue, rispettivamente in $X$ e $Y$ al variare di $t$ (efr. n. 4 e l'ipotesi (36)).

Tenuto conto dell'assoluta continuità delle funzioni $\phi_{j}\left(g_{i}(0 ; X, G(X))\right),(j=1, \ldots, m)$, è evidente allora che le funzioni

$$
\sum_{j=1}^{m} a_{i j}(X, G(X)) z_{j}^{(n)}(X, G(X))
$$


risultano assolutamente continue in $X$ in $\left(0, \beta_{h}\right)$, in virtù della $(41)$, per $i=1, \ldots, m$, con $i \neq h$.

Per $i=h$ più semplicemente dalla $i$-ma delle (21) segue subito, ove si tenga conto che $\left(X^{\prime}, Y^{\prime}\right)$ e $\left(X^{\prime \prime}, Y^{\prime \prime}\right)$ appartengono alla curva caratteristica $Y=g_{k}(X ; x, y)$,

$$
\begin{aligned}
& \left|\sum_{j=1}^{m} a_{i j}\left(X^{\prime}, Y^{\prime}\right) z_{j}^{(n)}\left(X^{\prime}, Y^{\prime}\right)-\sum_{j=1}^{m} a_{i j}\left(X^{\prime \prime}, Y^{\prime \prime}\right) z_{i}^{(n)}\left(X^{\prime \prime}, Y^{\prime \prime}\right)\right| \leqslant \\
& \leqslant \int_{X^{\prime}}^{X^{\prime \prime}}[(\mu(t)+\Lambda M(t)) U(t)+N(t)] d t<\int_{X^{\prime}}^{X^{n}} S(t) d t
\end{aligned}
$$

per ogni coppia di valori $X^{\prime}, X^{\prime \prime}$ di $\left(0, \beta_{h}\right)$, con $X^{\prime}<X^{\prime \prime}$, da cui risulta immediatamente l'assoluta continuità in $X$ della funzione $\sum_{j=1}^{m} a_{i j}\left(X, G(X) z_{j}^{(n)}(X, G(X))\right.$ in $\left(0, \beta_{h}\right)$.

Precisiamo che, preso $\varepsilon>0$, al numero $\delta$ (subordinato a $\varepsilon$ ) considerato all'inizio del capoverso si può subordinare un numero $\xi>0$, tale che, se $\left(X_{1}^{\prime} X_{1}^{\prime \prime}\right), \ldots,\left(X_{r}^{\prime}, X_{r}^{\prime \prime}\right)$ sono $r$ intervalli di $\left(0, \beta_{h}\right)$, con $X_{1}^{\prime}<X_{1}^{\prime \prime} \leqslant \ldots \leqslant X_{\psi}^{\prime}<X_{r}^{\prime \prime}$ \& $\sum\left|X_{k}^{\prime \prime}-X_{k}^{\prime}\right|<\xi$, risulti (cfr. n. 4)

$$
\sum_{k=1}^{r}\left|g_{i}\left(t ; X_{k}^{\prime}, Y_{k}^{\prime}\right)-g_{i}\left(t ; X_{k}^{\prime \prime}, Y_{k}^{\prime \prime}\right)\right|<\delta, \quad\left(0 \leqslant t \leqslant a_{0}\right)
$$

in virtù delle (26), (33), (36), (41), si ha allora ${ }^{(2 b i s)}$

$$
\begin{aligned}
& \sum_{k=1}^{r}\left|\sum_{j=1}^{m} a_{i j}\left(X_{k}^{\prime}, Y_{k}^{\prime}\right) z_{j}^{(n)}\left(X_{k}^{\prime}, Y_{k}^{\prime}\right)-\sum_{j=1}^{m} a_{i j}\left(X_{k}^{\prime \prime}, Y_{k}^{\prime \prime}\right) z_{j}^{(n)}\left(X_{k}^{\prime \prime}, Y_{k}^{\prime \prime}\right)\right|< \\
& \quad<\sum_{k=1}^{r} \int_{\boldsymbol{X}_{k}^{\prime}}^{x_{x_{k}}^{\prime \prime}}\left[S(t)+2 m \Lambda \lambda_{1} W M(t)\right] d t+\left[\frac{m B_{1}}{3}+m \int_{0}^{a}\left[\mu(t)+\Lambda M(t)+L_{\mathbf{1}}(t)\right) d t\right] \cdot \varepsilon .
\end{aligned}
$$

Allora, tenuto conto dell'assoluta continuità delle funzioni $a_{i i}(X, G(X))$, $(i, j=1, \ldots, m)$, risultano assolutamente continue anche le funzioni $z_{j}^{(n)}(X, G(X))$, cioè le funzioni $z_{j}^{(n)}\left(X, g_{h}(X ; x, y)\right),(j, h=1, \ldots, m),\left(0 \leqslant X \leqslant \beta_{h}(x, y)\right)$; in particolare esse sono a variazione limitata.

Indicata con

$$
\Omega(\psi(X))
$$

la variazione totale di una funzione $\psi=\psi(X)$, a variazione limitata nel proprio in-

$\left({ }^{12 b^{\prime}}\right.$ s) Dalla (33), tenendo conto che è $m B_{1} B_{2} \geqslant 1$ ( $\left.\mathrm{cfr} .\left({ }^{9}\right)\right)$, segue

$$
\begin{aligned}
\sum_{k=1}^{+} \sum_{j=1}^{m}\left|\phi_{j}\left(g_{i}\left(0 ; X_{k}^{\prime}, Y_{k}^{\prime}\right)\right)-\phi_{j}\left(g_{i}\left(0 ; X_{k}^{\prime \prime}, Y_{k}^{\prime \prime}\right)\right)\right| \leqslant & \\
& \leqslant m B_{1} B_{2} \sum_{i=1}^{n} \sum_{i=1}^{m} \sum_{j=1}^{m}\left|\phi_{j}\left(g_{i}\left(0 ; X_{k}^{\prime}, Y_{k}^{\prime}\right)\right)-\phi_{j}\left(g_{i}\left(0 ; X_{k}^{\prime \prime}, Y_{i k}^{\prime \prime}\right)\right)\right| \leqslant m \frac{\varepsilon}{3} .
\end{aligned}
$$


tervallo di definizione, segue dalle $(41),(42)$

$$
\begin{aligned}
\Omega\left(\sum_{j=1}^{m} a_{i j}(X, G(X)) z_{j}^{(n)}(X, G(X))\right) & \leqslant \int_{0}^{a_{0}} S(t) d t+m B_{1} V_{0}+ \\
& +m V \int_{0}^{\beta_{h}}\left[\mu(t)+A M(t)+L_{1}(t)\right] d t+2 m A \lambda_{1} W \int_{0}^{\beta_{h}} M(t) d t,
\end{aligned}
$$

avendo tenuto conto dell'ipotesi sulla variazione totale delle funzioni $z_{j}^{(n-1)}(x, y)$ rispetto a $y$, la quale non supera $V$, e del significato di $\nabla_{0}$ (cfr. n. 4).

Tenendo presente la posizione (28), ne segue, nell'intervallo dei valori $X$ per eui $(X, G(X))$ appartiene a $D$,

$$
\Omega\left(\sum_{j=1}^{m} a_{i j}(\ldots) z_{j}^{(i t)}(\ldots)\right) \leqslant F_{1}+m V \int_{0}^{a}\left[\mu(t)+\Lambda M(t)+L_{1}(t)\right] d t+2 m \Lambda \lambda_{1} W \int_{0}^{a} M(t) d t .
$$

Posto

$$
\sum_{j=1}^{m} a_{i j}(X, G(X)) z_{j}^{(n)}(X, G(X))=Z_{i}^{(n)}(X) \quad(i=1, \ldots, m)
$$

si ha

$$
z_{j}^{(n)}(X, G(X))=\sum_{i=1}^{m} \alpha_{i j}(X, G(X)) Z_{i}^{(n)}(X), \quad(j=1, \ldots, m) .
$$

Ricordando il significato di $F_{2}$ (cf., nota $\left({ }^{10}\right)$ ), si ha

$$
\begin{aligned}
& \Omega\left(z_{j}^{(n)}(X, G(X))\right) \leqslant m B_{1} \mathcal{U}\left(a_{0}\right) F_{2}+ \\
& \quad+m B_{2}\left\{F_{1}+m V \int_{0}^{a}\left[\mu(t)+A M(t)+L_{1}(t)\right] d t+2 m \Lambda \lambda_{1} W \int_{0}^{a} M(t) d t\right\},
\end{aligned}
$$

e infine, tenuto conto della posizione (29), si ottiene

$$
\begin{aligned}
\varrho\left(z_{j}^{(n)}(X, G(X))\right) \leqslant F+m^{2} B_{2} V \int_{0}^{a}\left[\mu(t)+\Lambda M(t)+L_{1}(t)\right] d t & + \\
& +2 m^{2} B_{2} \Lambda \lambda_{1} W \int_{0}^{a} M(t) d t .
\end{aligned}
$$

Essendo $W>F$ (cir. la (30)), si può prendere $a$, con $0<a \leqslant a_{0}$, sufficientemente piccolo affinchè sia verificata, oltre la (39), anche la

$$
F+m^{2} B_{2} V \int_{0}^{a}\left[\mu(t)+\Lambda M(t)+L_{1}(t)\right] d t+2 m^{2} B_{2} \Lambda \lambda_{1} W \int_{0}^{a} M(t) d t \leqslant W,
$$


da cui

$$
Q\left(z_{j}^{(m)}(X, G(X))\right) \leqslant W, \quad(j=1, \ldots, m)
$$

come si voleva dimostrare.

Osservazione. - Rileviamo che non è stata utilizzata l'assoluta continuità delle funzioni $z_{i}^{(n-1)}(X, G(X)),(j=1, \ldots, m)$, bensì la proprietà, meno restrittiva, che esse sono a variazione limitata; così come, nel successivo sottocapoverso iiii), viene utilizzata la proprietà che le funzioni $z_{j}^{(n)}(X, G(X))$ sono a variazione equilimitata per $n=1,2, \ldots$.

iii) Si dimostra facilmente che le funzioni

$$
z_{j}^{(n)}(x, y) \quad(j=1, \ldots, m)
$$

sono anche assolutamente continue in $x$.

Infatti, considerati due punti $\left(x^{\prime}, y\right),\left(x^{\prime \prime}, y\right)$, con $x^{\prime}<x^{\prime \prime}$, del campo $D$, tenuto conto delle (1), $\left(1^{\prime}\right),(4),(9),(22),(23),(25)$, con calcolo prevedibilmente del tutto analogo a quello sviluppato nel sottocapoverso ii), si ottiene

$$
\begin{aligned}
& \left|\sum_{j=1}^{m} a_{i j}\left(x^{\prime}, y\right) z_{j}^{(n)}\left(x^{\prime}, y\right)-\sum_{j=1}^{m} a_{i j}\left(x^{\prime \prime}, y\right) z_{j}^{(n)}\left(x^{\prime \prime}, y\right)\right|= \\
& =\mid \sum_{j=1}^{m} a_{i j}\left(0, g_{i}\left(0 ; x^{\prime}, y\right)\right) \phi_{i}\left(g_{i}\left(0 ; x^{\prime}, y\right)\right)+ \\
& +\int_{0}^{x^{\prime}}\left[\sum_{j=1}^{m} \frac{d a_{i j}\left(X, g_{i}\left(X ; x^{\prime}, y\right)\right)}{d X} z_{j}^{(n-1)}\left(X, g_{i}\left(X ; x^{\prime}, y\right)\right)+\right. \\
& \left.+f_{i}\left(X, g_{i}\left(X ; x^{\prime}, y\right), z_{1}^{(n-1)}(\ldots), \ldots, z_{m}^{(n-1)}(\ldots)\right)\right] d X-\sum_{j=1}^{m} a_{i j}\left(0, g_{i}\left(0 ; x^{\prime \prime}, y\right)\right) \phi_{i}\left(g_{i}\left(0 ; x^{\prime \prime}, y\right)\right)- \\
& -\int_{0}^{x^{\prime \prime}}\left[\sum_{j=1}^{m} \frac{d a_{i j}\left(X, g_{i}\left(X ; x^{\prime \prime}, y\right)\right)}{d X} z_{j}^{(n-1)}\left(X, g_{i}\left(X ; x^{\prime \prime}, y\right)\right)+\right. \\
& \left.+f_{i}\left(X, g_{i}\left(X ; x^{\prime \prime}, y\right), z_{1}^{(n-1)}(\ldots), \ldots, z_{m}^{(n-1)}(\ldots)\right)\right] d X \mid= \\
& =\mid \sum_{j=1}^{m}\left[a_{i j}\left(0, g_{i}\left(0 ; x^{\prime}, y\right)\right) \phi_{j}\left(g_{i}\left(0 ; x^{\prime}, y\right)\right)-a_{i j}\left(0, g_{i}\left(0 ; x^{\prime \prime}, y\right)\right) \phi_{j}\left(g_{i}\left(0 ; x^{\prime \prime}, y\right)\right)\right]+ \\
& +\sum_{j=1}^{m}\left[\left(a_{i j}\left(X, g_{i}\left(X ; x^{\prime}, y\right)\right)-a_{i j}\left(X, g_{i}\left(X ; x^{\prime \prime}, y\right)\right)\right) z_{j}^{(n-1)}\left(X, g_{i}\left(X ; x^{\prime}, y\right)\right)\right]_{X=0}^{X=x^{\prime}}- \\
& \quad-\int_{0}^{x^{\prime}} \sum_{j=1}^{m}\left[a_{i j}\left(X, g_{i}\left(X ; x^{\prime}, y\right)\right)-a_{i j}\left(X, g_{i}\left(X ; x^{\prime \prime}, y\right)\right)\right] \frac{d z_{j}^{(n-1)}\left(X, g_{i}\left(X ; x^{\prime}, y\right)\right)}{d X} d X+
\end{aligned}
$$




$$
\begin{aligned}
& +\int_{0}^{x^{\prime}}\left\{\sum_{i=1}^{m} \frac{d a_{i j}\left(X, g_{i}\left(X ; x^{\prime \prime}, y\right)\right)}{d X}\left[z_{j}^{(n-1)}\left(X, g_{i}\left(X ; x^{\prime}, y\right)\right)-z_{j}^{(n-1)}\left(X, g_{i}\left(X ; x^{\prime \prime}, y\right)\right)\right]+\right. \\
& \left.+f_{i}\left(X, g_{i}\left(X ; x^{\prime}, y\right)\right), z_{1}^{(n-1)}(\ldots), \ldots, z_{m}^{(n-1)}(\ldots)\right)- \\
& \left.-f_{i}\left(X, g_{i}\left(X ; x^{\prime \prime}, y\right), z_{1}^{(n-1)}(\ldots), \ldots, z_{m}^{(n-1)}(\ldots)\right)\right\} d X+ \\
& +\int_{x^{\prime}}^{x^{\prime \prime}}\left[\sum_{j=1}^{m} \frac{d a_{i j}\left(X, g_{i}\left(X ; x^{\prime \prime}, y\right)\right)}{d X} z_{j}^{(n-1)}\left(X, g_{i}\left(X ; x^{\prime \prime}, y\right)\right)+\right. \\
& \left.+f_{i}\left(X, g_{i}\left(X ; x^{\prime \prime}, y\right), z_{1}^{(n-1)}(\ldots), \ldots, z_{m}^{(n-1)}(\ldots)\right)\right] d X \mid \leqslant \\
& \leqslant B_{1} \sum_{j=1}^{m}\left|\phi_{i}\left(g_{i}\left(0 ; x^{\prime}, y\right)\right)-\phi_{j}\left(g_{i}\left(0 ; x^{\prime \prime}, y\right)\right)\right|+A น\left(x^{\prime}\right)\left|g_{i}\left(x^{\prime} ; x^{\prime \prime}, y\right)-y\right|+ \\
& +\int_{0}^{x^{\prime}}\left\{\Lambda\left|g_{i}\left(X ; x^{\prime}, y\right)-g_{i}\left(X ; x^{\prime \prime}, y\right)\right| \sum_{j=1}^{m_{3}} \frac{d z_{j}^{(n-1)}\left(X, g_{i}\left(X ; x^{\prime}, y\right)\right)}{d X}+\right. \\
& +\left[\mu(X)+\Lambda M(X)+L_{1}(X)\right] \sum_{j=1}^{m}\left|z_{j}^{(n-1)}\left(X, g_{i}\left(X ; x^{\prime}, y\right)\right)-z_{j}^{(n-1)}\left(X, g_{i}\left(X ; x^{\prime \prime}, y\right)\right)\right|+ \\
& \left.+L_{1}(X)\left|g_{i}\left(X ; x^{\prime}, y\right)-g_{i}\left(X ; x^{\prime \prime}, y\right)\right|\right\} d X+\int_{x^{\prime}}^{x^{\prime \prime}}[(\mu(X)+\Lambda M(X)) \cup(X)+N(X)] d X \leqslant \\
& \leqslant B_{1} \sum_{i=1}^{m}\left|\phi_{j}\left(g_{i}\left(0 ; x^{\prime}, y\right)\right)-\phi_{i}\left(g_{i}\left(0 ; x^{\prime \prime}, y\right)\right)\right|+ \\
& +\int_{x^{\prime}}^{x^{\prime \prime}}\left\{\left[\Lambda\left(_{0}\right)+\lambda_{1} \int_{0}^{a_{0}} L_{1}(\tau) d \tau\right] M(X)+[\mu(X)+\Lambda M(X)] \mathcal{u}^{\prime}(X)+N(X)\right\} d X+ \\
& +m \Lambda \lambda_{1} W \int_{x^{\prime}}^{x^{\prime \prime}} M(X) d X+ \\
& +\int_{0}^{x^{\prime}}\left[\mu(X)+\Lambda M(X)+L_{1}(X)\right] \sum_{j=1}^{m}\left|z_{j}^{(n-1)}\left(X, g_{i}\left(X ; x^{\prime}, y\right)\right)-z_{j}^{(n-1)}\left(X, g_{i}\left(X ; x^{\prime \prime}, y\right)\right)\right| d X,
\end{aligned}
$$

e infine, usufruendo della posizione (27), si ottiene

$$
\begin{aligned}
& \left|\sum_{j=1}^{m} a_{i j}\left(x^{\prime}, y\right) z_{j}^{(n)}\left(x^{\prime}, y\right)-\sum_{j=1}^{m} a_{i j}\left(x^{\prime \prime}, y\right) z_{j}^{(n)}\left(x^{\prime \prime}, y\right)\right| \leqslant \\
& \quad \leqslant \int_{x^{\prime}}^{x^{\prime \prime}} S(X) d X+m \Lambda \lambda_{1} W \int_{x^{\prime}}^{x^{\prime \prime}} M(X) d X+B_{1} \sum_{j=1}^{m_{m}}\left|\phi_{j}\left(g_{i}\left(0 ; x^{\prime}, y\right)\right)-\phi_{j}\left(g_{i}\left(0 ; x^{\prime \prime}, y\right)\right)\right|+ \\
& \quad+\int_{0}^{x^{\prime}}\left[\mu(X)+A M(X)+L_{1}(X)\right] \sum_{j=1}^{m_{m}}\left|z_{j}^{(n-1)}\left(X, g_{i}\left(X ; x^{\prime}, y\right)\right)-z_{j}^{(n-1)}\left(X, g_{i}\left(X ; x^{\prime \prime}, y\right)\right)\right| d X .
\end{aligned}
$$


Siano

$$
\left(x_{1}^{\prime}, x_{1}^{\prime \prime}\right), \quad\left(x_{2}^{\prime}, x_{2}^{\prime \prime}\right), \ldots,\left(x_{r}^{\prime}, x_{r}^{\prime \prime}\right)
$$

$r$ intervalli a due a due non sovrapposti, con $x_{1}^{\prime}<x_{1}^{\prime \prime} \leqslant x_{2}^{\prime}<\ldots \leqslant x_{r}^{\prime}<x_{r}^{\prime \prime}$, tali che i punti $\left(x_{k}^{\prime}, y\right),\left(x_{k}^{\prime \prime}, y\right),(k=1, \ldots, r)$, appartengano al campo $D$.

In virtì dell'ipotesi (6), a seconda che sia verificata la prima o la seconda delle (6) stesse, è

$$
g_{i}\left(X ; x_{k}^{\prime}, y\right) \geqslant g_{i}\left(X ; x_{k}^{\prime \prime}, y\right) \geqslant g_{i}\left(X ; x_{k+1}^{\prime}, y\right) \quad(k=1, \ldots, r-1)
$$

ovvero

$$
g_{i}\left(X ; x_{k}^{\prime}, y\right) \leqslant g_{i}\left(X ; x_{k}^{\prime \prime}, y\right) \leqslant g_{i}\left(X ; x_{k+1}^{\prime}, y\right)
$$

per ogni $X$ di $\left(0, a_{0}\right)$, vale a dire gli intervalli $\left(g_{i}\left(X ; x_{k}^{\prime}, y\right), g_{i}\left(X ; x_{k}^{\prime \prime}, y\right)\right),(k=1, \ldots, r)$, sono a due a due non sovrapposti.

Si ha allora

$$
\begin{aligned}
& \left(44^{\prime}\right) \quad \sum_{k=1}^{r}\left|\sum_{i=1}^{m} a_{i j}\left(x_{k}^{\prime}, y\right) z_{j}^{(n)}\left(x_{k}^{\prime}, y\right)-\sum_{j=1}^{m} a_{i j}\left(x_{k}^{n}, y\right) z_{k j}^{(n)}\left(x_{k}^{\prime \prime}, y\right)\right| \leqslant \\
& \leqslant \sum_{k=1}^{r} \int_{x_{k}^{\prime}}^{x_{k}^{n}}\left[S(X)+m \Lambda \lambda_{1} W M(X)\right] d X+
\end{aligned}
$$$$
+B_{1} \sum_{k=1}^{n} \sum_{j=1}^{m}\left|\phi_{j}\left(g_{i}\left(0 ; x_{k}^{\prime}, y\right)\right)-\phi_{j}\left(g_{i}\left(0 ; x_{k}^{\prime \prime}, y\right)\right)\right|+
$$$$
+\sum_{k=1}^{r} \int_{0}^{z_{k}^{\prime}}\left[\mu(X)+A M(X)+L_{\mathbf{i}}(X)\right] \sum_{j=1}^{m}\left|z_{j}^{\prime n-1)}\left(X, g_{i}\left(X ; x_{k}^{\prime}, y\right)\right)-z_{j}^{(n-1)}\left(X, g_{i}\left(X ; z_{k}^{n}, y\right)\right)\right| d X \text {. }
$$

Sia $\varepsilon$ un numero positivo arbitrario e sia $\delta$ il numero positivo subordinato ad $\varepsilon$ all'inizio del presente capoverso (ofr. anche la (31)).

In virtù dell'assoluta continuità in $x$ delle funzioni $g_{i}(X ; x, y),(i=1, \ldots, m)$, (cfr. n. 2), al numero $\delta$ subordiniamo un numero positivo $\eta_{1}$, tale che, se è

$$
\sum_{k=1}^{r}\left|x_{k}^{\prime}-x_{k}^{\prime \prime}\right|<\eta_{1}
$$

sia

$$
\sum_{k=1}^{r}\left|g_{i}\left(X ; x_{k}^{\prime}, y\right)-g_{i}\left(X ; x_{k}^{\prime \prime}, y\right)\right|<\delta, \quad(i=1, \ldots, m)
$$

per ogni $X$ di $\left(0, a_{0}\right)$. 
Inoltre, per l'assoluta continuità in $\left(0, a_{0}\right)$ della funzione

$$
\int_{0}^{x}\left[S(X)+m \Lambda \lambda_{1} W M(X)\right] d X
$$

al numero $\varepsilon$ si può subordinare un numero positivo $\eta_{2}$, tale che, se è

$$
\sum_{k=1}^{r}\left|x_{k}^{\prime}-x_{k}^{\prime \prime}\right|<\eta_{2}
$$

sia

$$
\sum_{k=1}^{r} \int_{x_{k}^{\prime}}^{x_{k}^{n}}\left\{S(X)+m A \lambda_{1} W M(X)\right\} d X<\varepsilon .
$$

Sia $\eta$ il minore dei due numeri $\eta_{1}, \eta_{2}$. Allora, se ̀̀

$$
\sum_{k=1}^{r}\left|x_{k}^{\prime}-x_{k}^{\prime \prime}\right|<\eta
$$

in virtù delle $(33),(36)$ si ottiene dalla $\left(44^{\prime}\right)$

$$
\begin{aligned}
\sum_{k=1}^{r} \mid \sum_{j=1}^{m} a_{i j}\left(x_{k}^{\prime}, y\right) z_{j}^{\prime n)}\left(x_{k}^{\prime}, y\right) & -\sum_{i=1}^{m} a_{i j}\left(x_{k}^{\prime \prime}, y\right) z_{j}^{(n)}\left(x_{k}^{\prime \prime}, y\right) \mid \leqslant \\
& \leqslant\left[1+\frac{m B_{1}}{3}+m \int_{0}^{a_{0}}\left(\mu(X)+A M(X)+L_{1}(X)\right) d X\right] \cdot \varepsilon .
\end{aligned}
$$

Dunque le funzioni

$$
\sum_{j=1}^{m} a_{i j}(x, y) z_{j}^{(n)}(x, y) \quad(i=1, \ldots, m)
$$

sono assolutamente continue in $x$; inoltre, essendo le (45), (46) indipendenti da $y$, esse risultano equiassolutamente continue in $x$ al variare di $y$ in $\left(-b_{0}, b_{0}\right)$.

In virtù dell'equiassoluta continuità in $x$, al variare di $y$, delle funzioni $a_{i j}(x, y)$, $(i, j=1, \ldots, m)$, ne segue (cfr. $\left.\left(1^{\prime \prime}\right)\right)$ l'equiassoluta continuità in $x$, al variare di $y$, delle funzioni $z_{j}^{(n)}(x, y),(j=1, \ldots, m)$, nel campo $D$.

è quindi

iiii) verifichiamo che per le funzioni $z_{j}^{(0)}(x, y),(j=1, \ldots, m)$, vale la $(36)$ ed

$$
V_{0}<V
$$

e inoltre

$$
\nabla_{0}<W
$$

Infatti per $X=x$ la (33) diviene

$$
\sum_{k=1}^{r} B_{1} B_{2} m \sum_{j=1}^{m n}\left|\phi_{j}\left(y_{k}^{\prime}\right)-\phi_{j}\left(y_{k}^{\prime \prime}\right)\right|<\frac{\varepsilon}{3}
$$


se è $\sum_{k=1}^{r}\left|y_{k}^{\prime}-y_{k}^{\prime \prime}\right|<\delta$; essendo (cfr. $\left.\left(^{9}\right)\right) m B_{1} B_{2} \geqslant 1$, per ogni $j_{0}$, con $j_{0}=1, \ldots, m$, è anche

$$
\sum_{k=1}^{r}\left|\phi_{j_{0}}\left(y_{k}^{\prime}\right)-\phi_{j_{0}}\left(y_{k}^{\prime \prime}\right)\right| \leqslant \sum_{k=1}^{r} \sum_{j=1}^{m}\left|\phi_{j}\left(y_{k}^{\prime}\right)-\phi_{j}\left(y_{k}^{\prime \prime}\right)\right|<\frac{\varepsilon}{3}<\varepsilon,
$$

e infine, fissato $\varepsilon=\varepsilon_{0}$,

$$
V_{0}<N_{0} \varepsilon_{0}=V
$$

Proviamo ora che ̀̀ $V_{0}<W$.

Infatti dalla (29) risulta $F>V_{0}$, e infine dalla (30), $W>F>V_{0}$.

Osserviamo che quest'ultima diseguaglianza implica che la variazione totale della funzione $\phi_{i}\left(g_{i}(X ; x, y)\right)$ rispetto a $X$ in $\left(0, \beta_{i}\right)$, la quale non supera la variazione totale della funzione $\phi_{j}(y)$ in $\left(-b_{0}, b_{0}\right)$ e quindi non supera $V_{0}$, è inferiore a $W$ : ciò vale per $i, j=1, \ldots, m$.

E provato pertanto che per le funzioni $z_{j}^{(0)}(x, y)=\phi_{j}(y)$ valgono le ipotesi formulate all'inizio del capoverso sulle funzioni $z_{j}^{(n-1)}(x, y),(j=1, \ldots, m)$.

Tenuto conto dell'osservazione del capoverso $e$ ), è dimostrato che le funzioni $z_{j}^{(n)}(x, y),(j=1, \ldots, m)$, costituiscono, per $n=1,2, \ldots$, una successione di funzioni equilimitate (cfr. la (25)) e equicontinue, le quali in corrispondenza ad ogni $x d i(0, a)$ sono equiassolutamente continue in $y$ in $I_{x}$ e in corrispondenza ad ogni $y d i\left(-b_{0}, b_{0}\right)$ sono equiassolutamente continue in $x$ nell'intervallo corrispondente (contenuto in $I_{y}$ ): valgono infatti le $\left(25^{\prime}\right),(36),(46)$, per $n=1,2, \ldots$.

e) Convergenza delle approssimazioni suocessive. Le funzioni $z_{j}^{(n)}(x, y),(j=1, \ldots, m)$, $(n=1,2, \ldots)$, convergono uniformemente nel campo $D$ a funzioni continue $z_{j}(x, y)$, $(j=1, \ldots, m)$, le quali risultano assolutamente continue in $y$, fissato $x$, e in $x$, fissato $y$. Più precisamente esse risultano equiassolutamente continue in $y$, al variare $d i x$, equiassolutamente continue in $x$, al variare di $y$.

Si osserva inoltre che le funzioni $z_{j}\left(X, g_{i}(X ; x, y)\right),(i, j=1, \ldots, m)$, risultano assolutamente continue in $X$ in $\left(0, \beta_{i}\right)$.

Le funzioni $z_{j}(x, y),(j=1, \ldots, m)$, soddisfano il sistema (10) nel campo $D$.

Poniamo nel campo $D$, per $n=1,2,3, \ldots$

$$
\omega_{n}(x)=\sup _{0 \leqslant X \leqslant x} \sup _{-b_{3}+\int_{0}^{z} M(t) d t \leqslant y \leqslant b_{0}-\int_{0}^{x} M(t) d t} \sum_{i=1}^{m}\left|z_{j}^{(n)}(X, y)-z_{j}^{(n-1)}(X, y)\right| .
$$

Dalle (21), a partire da $n=2$, scrivendo $X$ al posto di $x$ e $t$ al posto di $X$, si ottiene

$$
\begin{aligned}
& z_{j}^{(n)}(X, y)-z_{j}^{(n-1)}(X, y)=\sum_{s=1}^{m} \alpha_{i j}(x, y) \int_{0}^{X}\left\{\sum_{s=1}^{m} \frac{d a_{i s}\left(t, g_{i}(t ; x, y)\right)}{d t} .\right. \\
& \cdot\left[z_{s}^{(n-1)}\left(t, g_{i}(\ldots)\right)-z_{s}^{(n-2)}\left(t, g_{i}(. .)\right)\right]+f_{i}\left(t, g_{i}(\ldots), z_{1}^{(n-1)}(\ldots), \ldots, z_{m}^{(n-1)}(\ldots)\right)- \\
& \left.\quad \quad-f_{i}\left(t, g_{i}(\ldots), z_{1}^{(n-2)}(\ldots), \ldots, z_{m}^{(n-2)}(\ldots)\right)\right\} d t, \quad(j=1, \ldots, m),
\end{aligned}
$$


da cui

$$
\begin{aligned}
& \left|z_{j}^{\{n\}}(X, y)-z_{j}^{(n-1)}(X, y)\right| \leqslant \\
& \quad \leqslant B_{2} \sum_{i=1}^{m} \int_{0}^{X}\left(\mu(t)+\Lambda M(t)+L_{1}(t)\right) \sum_{s=1}^{m}\left|z_{s}^{(n-1)}\left(t, g_{i}(\ldots)\right)-z_{s}^{(n-2)}\left(t, g_{i}(\ldots)\right)\right| d t \leqslant \\
& \quad \leqslant m B_{2} \int_{0}^{X}\left(\mu(t)+\Lambda M(t)+L_{1}(t)\right) \omega_{n-1}(t) d t .
\end{aligned}
$$

Posto

$$
m^{2} B_{2}\left(\mu(t)+\Lambda M(t)+L_{1}(t)\right)=\gamma(t)
$$

si ottiene

$$
\sum_{j=1}^{m}\left|z_{j}^{(n)}(X, y)-z_{j}^{(n-1)}(X, y)\right| \leqslant \int_{0}^{X} \gamma(t) \omega_{n-1}(t) d t,
$$

la quale vale per ogni $y$ di $I_{X}$.

Consideriamo l'intervallo $0 \leqslant X \leqslant x$, per $0<x \leqslant a$.

E evidentemente

$$
\begin{aligned}
\sum_{j=1}^{m}\left|z_{j}^{(n)}(X, y)-z_{j}^{(n-1)}(X, y)\right| \leqslant \int_{0}^{\infty} \gamma(t) \omega_{n-1}(t) d t & \\
& \left(-b_{0}+\int_{0}^{X} M(t) d t \leqslant y \leqslant b_{0}-\int_{0}^{X} M(t) d t, 0 \leqslant X \leqslant x\right),
\end{aligned}
$$

e quindi anche

$$
\omega_{n}(x) \leqslant \int_{0}^{x} \gamma(t) \omega_{n-1}(t) d t, \quad(0 \leqslant \infty \leqslant a),(n=2,3, \ldots) .
$$

Essendo, per le (47), $\left(25^{\prime}\right)$ (considerate per $\left.n=1\right)$, (22)

$$
\omega_{1}(x) \leqslant \mathcal{U}(x)+C \leqslant \mathcal{U}(a)+C \quad(0 \leqslant x \leqslant a),
$$

posto

$$
\mathcal{u}(a)+C=C_{0},
$$

si hat

$$
\omega_{2}(x) \leqslant \sigma_{0} \int_{0}^{x} \gamma(t) d t \quad(0 \leqslant x \leqslant a)
$$

e successivamente

$$
\omega_{3}(x) \leqslant C_{0} \int_{0}^{x} \gamma(t)\left(\int_{0}^{t} \gamma(\tau) d \tau\right) d t=C_{0} \frac{\left(\int_{0}^{x} \gamma(\tau) d \tau\right)^{2}}{2}, \quad(0 \leqslant x \leqslant a) .
$$

11 - Annali di Maternatica 
Supposto ora che valga la

$$
\omega_{n-1}(x) \leqslant C_{0} \frac{\left(\int_{0}^{x} \gamma(\tau) d \tau\right)^{n-2}}{(n-2) !} \quad(0 \leqslant x \leqslant a),
$$

dimostriamo che, in virtù della (48), è anche

$$
\omega_{n}(x) \leqslant C_{0} \frac{\left(\int_{a}^{x} \gamma(\tau) d \tau\right)^{n-1}}{(n-1) !} \quad(0 \leqslant x \leqslant a) .
$$

Infatti ̀̀

$$
\omega_{n}(x) \leqslant \int_{0}^{x} \gamma(t) \omega_{n-1}(t) d t \leqslant C_{0} \int_{0}^{\infty} \gamma(t) \frac{\left(\int_{0}^{t} \gamma(\tau) d \tau\right)^{n-2}}{(n-2) !} d t=C_{0} \frac{\left(\int_{0}^{x} \gamma(\tau) d \tau\right)^{n-1}}{(n-1) !} \quad(0 \leqslant x \leqslant a) .
$$

Allora, in virtù del principio di induzione, per $n=2,3, \ldots$ è in $(0, a)$

$$
\omega_{n}(x) \leqslant C_{0} \frac{\left(\int_{0}^{x} \gamma(t) d t\right)^{n-1}}{(n-1) !} \leqslant C_{0} \frac{\left(\int_{0}^{a} \gamma(t) d t\right)^{n-1}}{(n-1) !} .
$$

Dalla convergenza della serie numerica

$$
\sum_{n=1}^{\infty} \frac{\left(\int_{0}^{a} \gamma(t) d t\right)^{n}}{n !}
$$

segue in modo noto la convergenza uniforme delle funzioni $z_{j}^{(n)}(x, y),(j=1, \ldots, m)$, a funzioni continue $z_{i}(x, y),(j=1, \ldots, m)$, in tutto il campo $D$.

Le funzioni

$$
z_{j}(x, y)=\lim _{n \rightarrow \infty} z_{j}^{(n)}(x, y)
$$

soddisfano nel campo $D$ il sistema (10) e appartengono alla classe funzionale precisata all'inizio del capoverso: valgono infatti per esse le (36), $(46),\left(42^{\prime}\right)$, le quali sono indipendenti rispettivamente da $x, y,(x, y)$.

f) Le funzioni $z_{j}(x, y),(j=1, \ldots, m)$, soddisfano in quasi tutto il campo $D$ il sistema (I) e inoltre le condizioni (II) (cfr. n. 3).

\section{6. - Estensione dell'esistenza della soluzione a tutto il campo $D_{0}$.}

a) Valgano ancora le ipotesi formulate nel n. 5, a).

Nella dimostrazione del teorema di esistenza sviluppata nel n. 5, abbiamo seguito questo ordine (cfr. capoverso $d)$ ) : abbiamo preso una costante positiva $W$, soddisfacente la $W>F$ (dove $F$ è funzione di $V_{0}$ ), e abbiamo poi determinato $a$ in modo 
da soddisfare, oltre alla

$$
m^{2} B_{2} N_{\lambda_{1}} \int_{0}^{a}\left(\mu(t)+\Lambda M(t)+L_{1}(t)\right) d t \leqslant \frac{1}{3}
$$

la

$$
F+m^{2} B_{2} V \int_{0}^{a}\left(\mu(t)+\Lambda M(t)+L_{1}(t)\right) d t+2 m^{2} B_{2} \Lambda \lambda_{1} W \int_{0}^{a} M(t) d t \leqslant W,
$$

dove si può avere anche l'uguaglianza.

Posto per brevità

$$
\begin{aligned}
& m^{2} B_{2}\left(\mu(t)+\Lambda M(t)+L_{1}(t)\right)=\gamma_{1}(t) \\
& 2 m^{2} B_{2} \Lambda \lambda_{1} M(t)=\gamma_{2}(t)
\end{aligned}
$$

la (44) si scrive nella forma

$$
F^{\prime}+V \int_{0}^{a} \gamma_{1}(t) d t+W \int_{0}^{a} \gamma_{2}(t) d t \leqslant W
$$

Ragioniamo ora in modo diverso, rispetto al capoverso $d$ ) del n. 5 .

Sia $a_{1}$ un numero, non superiore ad $a_{0}$, tale che per qualunque coppia $x^{\prime}, x^{\prime \prime}$ di $\left(0, a_{0}\right)$, con $\left|x^{\prime}-x^{\prime \prime}\right| \leqslant a_{1}$, sia

$$
\left|\int_{x^{\prime}}^{x^{\prime \prime}} \gamma_{2}(t) d t\right| \leqslant \frac{1}{2}
$$

Sia poi $a_{2}$ un numero, non superiore ad $a_{0}$, tale che per qualunque coppia $x^{\prime}, x^{\prime \prime}$ di $\left(0, a_{0}\right)$, con $\left|x^{\prime}-x^{\prime \prime}\right| \leqslant a_{2}$, sia

$$
m^{2} B_{2} N_{\lambda_{1}}\left|\int_{x^{\prime}}^{x^{n}}\left(\mu(t)+\Lambda M(t)+L_{1}(t)\right) d t\right| \leqslant \frac{1}{3} ;
$$

sia $a^{*}$ il più piccolo dei due numeri $a_{1}, a_{2}\left({ }^{13}\right)$.

Sia $W$ il numero che soddisfa la relazione

$$
F^{\prime}+V \int_{0}^{a^{*}} \gamma_{1}(t) d t+W \int_{0}^{a^{*}} \gamma_{2}(t) d t=W
$$

(13) In particolare si ha

$$
\begin{gathered}
m^{2} B_{2} N_{\lambda_{1}} \int_{0}^{a^{*}}\left(\mu(t)+\Lambda M(t)+I_{1}(t)\right) d t \leqslant \frac{1}{3}, \\
\int_{0}^{a^{*}} \gamma_{2}(t) d t \leqslant \frac{1}{2} .
\end{gathered}
$$


dopodichè la dimostrazione dell'esistenza della soluzione del sistema (10) rimane inalterata, nel campo (cfr. (39), (44))

$D^{*}: \quad 0 \leqslant x \leqslant a^{*}, \quad-b_{0}+\int_{0}^{x} M(t) d t \leqslant y \leqslant b_{0}-\int_{0}^{\infty} M(t) d t$.

Osserviamo ora che $a_{1}, a_{2}$, e quindi $a^{*}$, dipendono soltanto dalle funzioni $a_{i j}(\ldots)$ $\varrho_{i}(\ldots), f_{i}(\ldots),(i, j=1, \ldots, m)$, ehe figurano nel sistema $(\mathrm{I})$; sono indipendenti invece dai dati $\phi_{s}(y),(j=1, \ldots, m)\left({ }^{14}\right)$.

Allora, se a partire dai valori assunti dalla soluzione per $x=a^{*}$

$$
z_{1}\left(a^{*}, y\right), \quad z_{2}\left(a^{*}, y\right), \ldots, z_{m}\left(a^{*}, y\right), \quad\left(-b_{0}+\int_{0}^{x} M(t) d t \leqslant y \leqslant b_{0}-\int_{0}^{\infty} M(t) d t\right),
$$

si ripete la dimostrazione del teorema di esistenza, si trova che nel campo

$$
a^{*} \leqslant x \leqslant 2 a^{*}, \quad-b_{0}+\int_{0}^{a} M(t) d t \leqslant y \leqslant b_{0}-\int_{0}^{a} M(t) d t
$$

esiste una soluzione del sistema (I), appartenente al campo funzionale richiesto, la quale assume per $x=a^{*}$ i valori $\left(50^{\prime}\right)$.

E chiaro che ripetendo un numero finito di volte tale estensione del campo, si ottiene l'esistenza della soluzione del sistema (I) sotto le condizioni (II) nell'intero campo $D_{0}$.

b) Quanto abbiamo dimostrato nel capoverso $a$ ) è valido se in tutto il campo $D_{0}$ per ogni $i,(i=1, \ldots, m)$, vale una (fissata) delle (6), e per ogni coppia di valori diversi $i, h(i, h=1, \ldots, m ; i \neq h)$ vale una (fissata) delle (7).

Supponiamo ora invece, come nell'enunciato del Teorema del n. 1, che l'intervallo $\left(0, a_{0}\right)$ si possa decomporre in un numero finito $r$ di intervalli

$$
\left(0, a^{(1)}\right), \quad\left(a^{(1)}, a^{(2)}\right), \ldots,\left(a^{(r-1)}, a^{(r)}\right)
$$

tali che, in ciascuno dei campi $D^{(k)}$

$D^{(k)}: \quad a^{(k-1)} \leqslant x \leqslant a^{(k)}, \quad-b_{0}+\int_{0}^{x} M(t) d t \leqslant y \leqslant b_{0}-\int_{0}^{x} M(t) d t$,

per ogni $i,(i=1, \ldots, m)$, valga una delle $(6)$ e per ogni coppia di valori $i, h$, $(i, h=1, \ldots, m ; i \neq h)$, valga una delle $(7)$.

Allora la soluzione $z_{1}(x, y), \ldots, z_{m}(x, y)$, costruita nei nn. 5,6 , esiste senz'altro nel campo $D^{(1)}$, e, successivamente, nei campi $D^{(2)}, \ldots, D^{(r)}$, vale a dire in tutto il campo $D_{0}$.

$\left.{ }^{(14}\right)$ Il numero $a_{1}$ dipende soltanto da $m, B_{2}, \Lambda, \lambda_{1}, M(t): a_{2}$ da $m, B_{2}, \lambda_{1}, \mu(t), \Lambda, M(t), L_{1}(t)$. 
Osservazione. - Rileviamo esplicitamente (cfr. e)) che la soluzione costruita nel campo $D_{0}$ appartiene al campo funzionale precisato nell'enunciato del teorema, e inoltre (cfr. le $\left(42^{\prime}\right)$ ) le funzioni $z_{j}(x, y),(j=1, \ldots, m)$, risultano assolutamente continue lungo le caratteristiche del sistema (I).

\section{7. - Unicità della soluzione.}

È immediata l'unicità della soluzione nel campo delle funzioni $z_{j}(x, y),(j=1, \ldots, m)$, le quali, oltre ad essere assolutamente continue rispetto a ciascuna variabile $x, y$, separatamente, sono anche assolutamente continue lungo una qualunque curva caratteristica $Y=g_{i}(X ; x, y)$, vale a dire sono tali che risultano assolutamente continue rispetto a $X$ in $\left(0, \beta_{i}\right)$ le funzioni $z_{j}\left(X, g_{i}(X ; x, y)\right),(i, j=1, \ldots, m)$.

Infatti una soluzione del sistema (I) sotto le condizioni (II) nel campo $D_{0}$, appartenente al suddetto campo funzionale, soddisfa in tutto $D_{0}$ il sistema (10) (cfr. n. $3, b)$ ) : e l'unicità della solnzione del sistema (10) è subito provata. Infatti se $z_{j}(x, y), z_{j}^{*}(x, y),(j=1, \ldots, m)$, sono due soluzioni del sistema (10), soddisfacenti le ipotesi suddette, si ha

$$
\begin{array}{r}
\sum_{j=1}^{m} a_{i j}(x, y)\left[z_{j}(x, y)-z_{j}^{*}(x, y)\right]=\int_{0}^{x}\left\{\sum_{j=1}^{m} \frac{d x_{i j}\left(X, g_{i}(X ; x, y)\right)}{d X}\left[z_{j}\left(X, g_{i}(\ldots)\right)-z_{j}^{*}\left(X, g_{i}(\ldots)\right)\right]+\right. \\
\left.+f_{i}\left(X, g_{i}(\ldots), z_{1}\left(X, g_{i}(\ldots)\right), \ldots, z_{m}(\ldots)\right)-f_{i}\left(X, g_{i}(\ldots), z_{1}^{*}(\ldots), \ldots, z_{m}^{*}(\ldots)\right)\right\} d X, \\
(i=1, \ldots, m),
\end{array}
$$

da cui

$$
\begin{array}{r}
\left|z_{j}(x, y)-z_{i}^{*}(x, y)\right| \leqslant B_{2} \int_{0}^{x}\left[\mu(X)+A M(X)+L_{1}(X)\right] \sum_{i=1}^{m} \sum_{s=1}^{m}\left|z_{s}\left(X, g_{i}(\ldots)\right)-z_{s}^{*}\left(X, g_{i}(\ldots)\right)\right| d X, \\
(j=1, \ldots, m) .
\end{array}
$$

Ne segue $\left({ }^{15}\right)$ in tutto $D_{0}$

$$
z_{j}(x, y)=z_{j}^{*}(x, y), \quad(j=1, \ldots, m)
$$

(15) Cfr. M. Cinquini Cibrario - S. Crnquini, Equazioni a devivate parziali di tipo iperbolico, Monografie Matematiche del C.N.R., no. 12, Edizioni Cremonesi, Roma (1964); cap. IV, $\$ 2$, n. $9 \delta$ ), pp. 352-353; efr. in particolare la $(80)$.

Viene utilizzato il lemma di Gronwall generalizzato, efr. G. SANsone - R. Contr, Equa* zioni differenziali non lineari, Monografie Matematiche del C.N.R., Edizioni Cremonesi, Roma (1956), cap. I, \$ 2, n. 1, pp. 15-16, 


\section{$\$ 2$.}

\section{8. - Un esempio.}

Mettiamo in luce con un esempio che, se non vale la (6), un'eventuale soluzione del sistema (10) (efr. n. 3) può non appartenere alla classe delle funzioni assolutamente continue rispetto alle singole variabili $x, y$.

Consideriamo nell'intervallo $(0,2 / \pi)$ la funzione $\sigma(t)$ cosi definita:

$$
\sigma(0)=0, \quad \sigma(t)=t^{2} \operatorname{sen} \frac{1}{t} \quad\left(0<t \leqslant \frac{2}{\pi}\right) .
$$

Nel campo

$$
0 \leqslant X \leqslant \frac{2}{\pi}, \quad 0 \leqslant x \leqslant \frac{2}{\pi}, \quad-\infty<y<+\infty
$$

consideriamo la funzione

$$
g(X ; x, y)=y+\int_{x}^{x} \sigma^{\prime}(t) d t
$$

cioè

$$
g(X ; x, y)=y-x^{2} \operatorname{sen} \frac{1}{x}+X^{2} \operatorname{sen} \frac{1}{X}
$$

dove si intende che per $x$ (oppure $X$ ) nullo in luogo di $x^{2}$ sen $1 / x\left(X^{2} \operatorname{sen} 1 / X\right)$ compaia zero.

Sia $f(x)$ una funzione quasi continua e integrabile in $(0,2 / \pi)$.

Considerata la funzione

$$
\phi(y)=\sqrt{|y|} \quad(-\infty<y<+\infty)
$$

la quale è assolutamente continua $\left(^{(6)}\right.$ in ogni intervallo, prendiamo in esame l'equazione (17)

$$
z(x, y)=\phi(g(0 ; x, y))+\int_{0}^{x} f(X) d X
$$

(16) La frunzione $\sqrt{|y|}$ è la funzione integrale della funzione $p(y)$ così definita:

$$
\varphi(y)=\frac{1}{2 \sqrt{y}} \quad \text { per } y>0, \quad \varphi(y)=-\frac{1}{2 \sqrt{-y}} \quad \text { per } y<0,
$$

la quale è quasi continua e integrabile secondo Lebesgue, con $\int_{0}^{y} \varphi(Y) d Y=\sqrt{|y|}$.

(17) Rileviamo che per l'equazione (51), la quale è un particolare sistema (10), con $m=1$, $a_{1}(\ldots)=1, \varrho_{1}(\ldots)=\sigma^{\prime}(x), f_{1}(\ldots)=f(x)$, valgono tutte le ipotesi del n. 3 (cfr. n. 1) tranne la (6). Infattị la funzione $\sigma^{\prime}(x)$ cambia segno infinite volte in $(0,2 \pi)$. 
Si ha

$$
z(x, y)=\sqrt{\left|y-x^{2} \operatorname{sen} \frac{1}{x}\right|}+\int_{0}^{x} f(X) d X,
$$

e, per esempio per $y=0$,

$$
z(x, 0)=x \sqrt{\operatorname{sen} \frac{1}{x} \mid}+\int_{0}^{x} f(X) d X ;
$$

la funzione $z(x, 0)$ non è a variazione limitata in $(0,2 / \pi)$.

Infatti, considerata una suddivisione di $(0,2 / \pi)$ in $2 k+1$ parti mediante $i$ punti

$$
\begin{aligned}
& x_{0}=0, \quad x_{1}=\frac{1}{(2 k+1) \pi / 2}, \quad x_{2}=\frac{1}{2 k \pi / 2}, \quad x_{3}=\frac{1}{(2 k-1) \pi / 2}, \ldots, \\
& x_{2 s}=\frac{1}{(2 k-2 s+2) \pi / 2}, \quad x_{2 s+1}=\frac{1}{(2 k-28+1) \pi / 2}, \ldots, \\
& x_{2 k-1}=\frac{1}{3 \pi / 2}, \quad x_{2 k}=\frac{1}{2 \pi / 2}, \quad x_{2 k+1}=\frac{1}{\pi / 2},
\end{aligned}
$$

si ha, se $f(x) \geqslant 0$,

$$
\begin{aligned}
\sum_{s=0}^{k}\left|z\left(x_{2 s+1}, 0\right)-z\left(x_{2 s}, 0\right)\right|=\sum_{s=0}^{k} \mid x_{2 s+1} \sqrt{\left|\operatorname{sen} \frac{1}{x_{2 s+1}}\right|} & -x_{2 s} \sqrt{\left|\operatorname{sen} \frac{1}{x_{2 s}}\right|}+\int_{x_{k s}}^{x_{2 s+1}} f(X) d X \mid \geqslant \\
& \geqslant \frac{2}{\pi}\left(\frac{1}{2 k+1}+\frac{1}{2 k-1}+\ldots+\frac{1}{3}+1\right) .
\end{aligned}
$$

Essendo $\lim _{k \rightarrow+\infty}\left(1+\frac{1}{3}+\ldots+1 /(2 k-1)+1 /(2 k+1)\right)=+\infty$, la funzione $z(x, 0)$ non è a variazione limitata in $(0,2 / \pi)$.

Osservazione. - Più in generale, se le funzioni $\phi_{j}\left(g_{i}(0 ; x, y)\right)$ non sono assolutamente continue rispetto alle variabili $x, y$ separatamente, un'eventuale soluzione $z_{j}(x, y),(j=1, \ldots, m)$, del sistema (10) può non essere costituita da funzioni assolutamente continue rispetto a $x$ e $y$ separatamente.

Non lo è certamente se le funzioni $a_{i j}(0, y)$ sono per esempio nulle per $i \neq j$ e se le funzioni $f_{i}(\ldots)$ sono indipendenti da $z_{1}, \ldots, z_{m}$, come nell'esempio dato.

Si può provare che, se una soluzione del sistema (10), per il quale valgano le ipotesi del n. 1, appartiene alla classe delle funzioni equiassolutamente continue in $y$ al variare di $x$, assolutamente continue in $x$ e assolutamente continue lungo le caratteristiche (come avviene per la soluzione costruita nel $\S 1$ ) e se vale suddetta ipotesi sulle funzioni $a_{i j}(0, y),(i \neq j)$, necessariamente le funzioni $\phi_{i}\left(g_{i}(0 ; x, y)\right)$ sono assolutamente continue nelle singole variabili, 


\section{9. - Un ulteriore esempio.}

Rileviamo con un esempio che, se non valgono le (7), considerata un'eventuale soluzione $z_{j}(x, y),(j=1, \ldots, m)$, del sistema $(10)$, costituita da funzioni assolutamente continue rispetto alle singole variabili $x, y$, può mancare l'assoluta continuitè in $X$ delle funzioni $z_{j}\left(X, g_{i}(X ; x, y)\right)$.

Considerato un angolo $\alpha$, fissato tra 0 e $\pi / 4,0<\alpha<\pi / 4$, definiamo nell'intervallo $(0,1)$ la funzione $\varphi(\xi)$ nel seguente modo:

$$
\begin{array}{ll}
\varphi(\xi)=\left(\xi-\frac{1}{2}\right) \operatorname{tg} \alpha, & \left(\frac{1}{2} \leqslant \xi \leqslant 1\right), \\
\varphi(\xi)=-\left(\xi-\frac{1}{2}\right) \operatorname{cotg} \beta_{1}, & \left(\frac{1}{3} \leqslant \xi \leqslant \frac{1}{2}\right), \\
\varphi(\xi)=\left(\xi-\frac{1}{4}\right) \operatorname{tg} \alpha, & \left(\frac{1}{4} \leqslant \xi \leqslant \frac{1}{3}\right), \\
\varphi(\xi)=-\left(\xi-\frac{1}{4}\right) \operatorname{cotg} \beta_{2}, & \left(\frac{1}{5} \leqslant \xi \leqslant \frac{1}{4}\right), \\
. . . . . . & \\
\varphi(\xi)=\left(\xi-\frac{1}{2 n}\right) \operatorname{tg} \alpha, & \left(\frac{1}{2 n} \leqslant \xi \leqslant \frac{1}{2 n-1}\right), \\
\varphi(\xi)=-\left(\xi-\frac{1}{2 n}\right) \operatorname{cotg} \beta_{n}, & \left(\frac{1}{2 n+1} \leqslant \xi \leqslant \frac{1}{2 n}\right), \\
. \cdots . . . & \\
\varphi(0)=0, &
\end{array}
$$

dove $\beta_{1}, \beta_{2}, \ldots, \beta_{n}, \ldots$ sono gli angoli definiti nel modo che segue.

Siano $\xi, \zeta$ due assi cartesiani ortogonali.

Consideriamo sull'asse $\xi$ i punti di ascissa $1 / 2 n+2,1 / 2 n+1,1 / 2 n$; per il punto $P_{2 n+2}(1 / 2 n+2,0)$ (cfr. figura) tracciamo la retta $r_{n}$ che, orientata nel senso delle ascisse crescenti, forma con il semiasse delle $\xi$ positive l'angolo $\alpha$; consideriamo poi per il punto $H_{2 n+1}(1 / 2 n+1,0)$ la perpendicolare $p_{n}$ all'asse $\xi$, la quale interseca la retta $r_{n}$ in un punto, che indichiamo con $P_{2 n+1}$; congiungiamo tale punto con il punto $P_{2 n}(1 / 2 n, 0)$; chiamiamo $\left({ }^{18}\right) \beta_{n}$ l'angolo $H_{28+1} \widehat{P}_{2 n+1} P_{2 n}$.

Risulta $\left({ }^{19}\right) \pi / 4<\beta_{n}<\pi / 2$.

(18) Gli angoli $\beta_{n}$ sono presi positivi.

${ }^{\left({ }^{99}\right)} \mathrm{E}$ evidente infatti che è $\beta_{n}<\pi / 2$.
Inoltre, indicato con $\beta$ (costante rispetto a $n$ ) l'angolo $P_{2 n+2} \widehat{P}_{2 n+1} H_{2 n+1}$, e indicata con $d_{n}$ la misura del segmento $P_{2 n+1} H_{2 n+1}$, si ha

$$
\operatorname{tg} \beta=\left(\frac{1}{2 n+1}-\frac{1}{2 n+2}\right): d_{n}, \quad \operatorname{tg} \beta_{n}=\left(\frac{1}{2 n}-\frac{1}{2 n+1}\right): d_{n}
$$




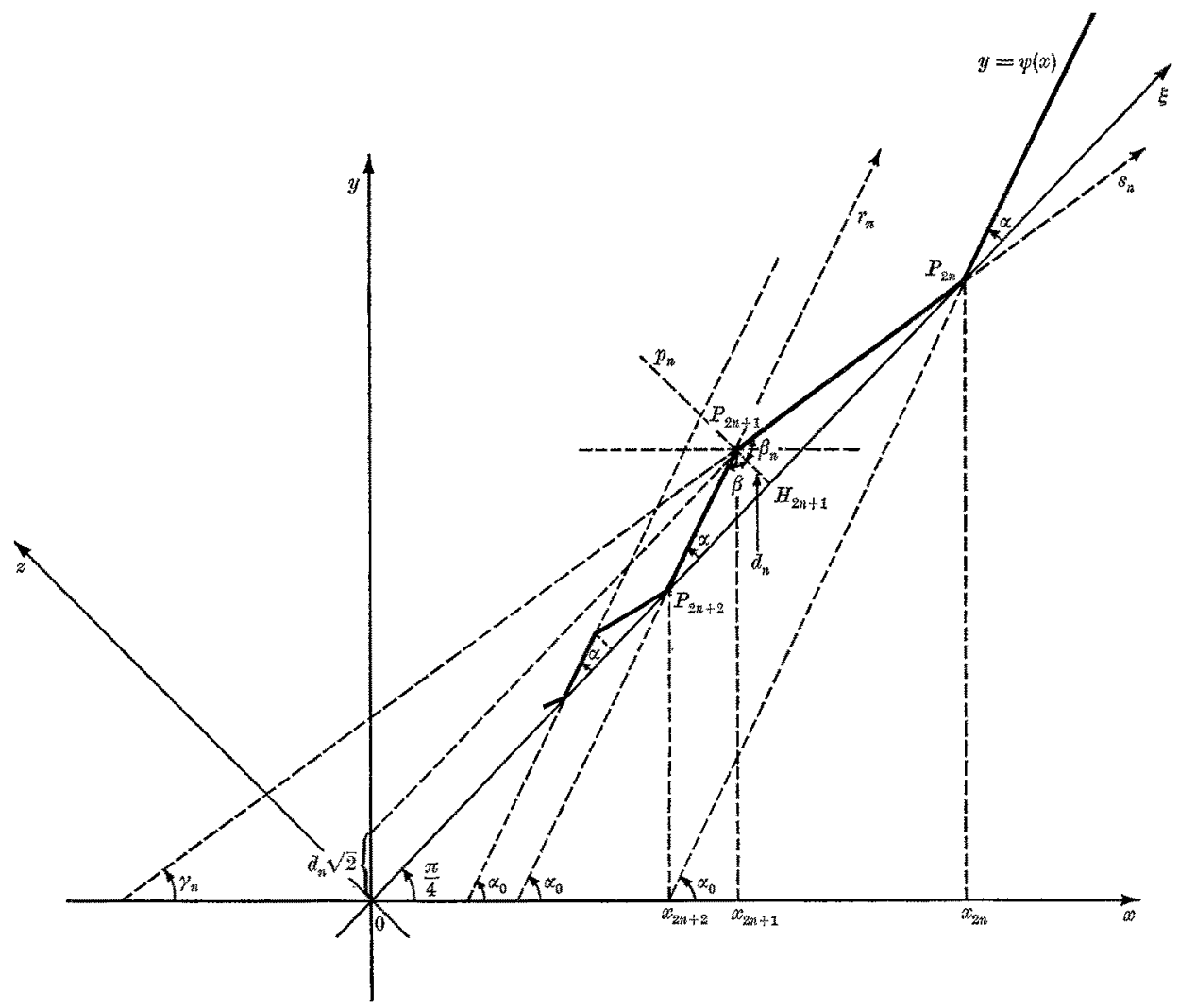

Osserviamo che la funzione $\zeta=\varphi(\xi)$ che abbiamo definito in $(0,1)$ in ogni intervallo $(1 /(n+1), 1 / n),(n=1,2, \ldots)$, ha come grafica un segmento, che ha direzione costante per $n$ dispari, direzione variabile con $n$ per $n$ pari.

Consideriamo ora la stessa curva $O$, di equazione

$$
\zeta=\varphi(\xi) \quad(0 \leqslant \xi \leqslant 1)
$$

riferita ad un nuovo sistema di assi cartesiani ortogonali $x, y$, aventi l'origine coincidente con quella del sistema $\xi$, $\zeta$, ruotati di $\pi / 4$ rispetto al sistema $\xi, \zeta$, precisamente tali che il semiasse delle $x$ positive debba ruotare di $\pi / 4$ nel senso delle rotazioni positive per sovrapporsi al semiasse delle $\xi$ positive.

ciò̀

da eui risulta

$$
\operatorname{tg} \beta=\frac{1}{(2 n+1)(2 n+2) d_{n}}, \quad \operatorname{tg} \beta_{n}=\frac{1}{2 n(2 n+1) d_{n}},
$$

e anche

$$
\operatorname{tg} \beta<\operatorname{tg} \beta_{n}
$$

$$
\beta<\beta_{n}
$$

Essendo $\beta+\alpha=\pi / 2$, da cui $\beta>\pi / 4$ (perchè è $\alpha<\pi / 4$ ), ne segue $\beta_{n}>\pi / 4$. 
Poichè l'angolo $\alpha$ è minore di $\pi / 4$ e gli angoli $\beta_{1}, \beta_{2}, \ldots, \beta_{n}, \ldots$ sono minori dì $\pi / 2$, ogni retta $x=k$, con $k$ costante, interseca ciascun segmento della curva $C$ in uno ed un sol punto, quando $k$ appartiene all'intervallo che ha per estremi le proiezioni degli estremi del segmento stesso; inoltre, per il modo in cui è stato scelto $\alpha$, compreso tra 0 e $\pi / 4$, risultando inoltre $\beta_{1}, \beta_{2}, \ldots, \beta_{n}, \ldots$, compresi tra $\pi / 4$ e $\pi / 2$, ogni retta $x=k$ interseca la curva $C$ in uno ed un sol punto, per $0 \leqslant k \leqslant 1 / \sqrt{2}$.

Indichiamo con

$$
y=\psi(x) \quad\left(0 \leqslant x \leqslant \frac{1}{\sqrt{2}}\right)
$$

l'equazione della curva $C$ rispetto al nuovo sistema di riferimento.

La funzione $\psi(x)$ risulta crescente.

Osserviamo che, indicata con $x_{n}$ l'ascissa del punto $P_{n},(n=1,2, \ldots)$, in ciascun intervallo $\left(x_{n+1}, x_{n}\right)$ la curva coincide con un segmento, avente direzione costante se $n$ è dispari, variabile con $n$ se $n$ è pari.

Per $n$ dispari la direzione è quella comune alle rette $r_{n}$, le quali formano con l'asse $x$ l'angolo $\alpha_{0}$, con $\alpha_{0}=\alpha+\pi / 4$. Risulta $\pi / 4<\alpha_{0}<\pi / 2$.

Per $n$ pari la direzione è quella della retta $s_{n}$ congiungente i punti $P_{2 n+1}, P_{2 n}$, la quale, formando con le rette $\xi=$ cost l'angolo $\beta_{n}$, con $\pi / 4<\beta_{n}<\pi / 2$, con l'asse $x$ forma, se orientata nel senso delle ascisse $x$ crescenti, l'angolo $\gamma_{n}$, con $0<\gamma_{n}<\pi / 4$.

La derivata $\psi^{\prime}(x)$ è costante a tratti e limitata, quindi integrabile.

Assumiamo

$$
\varrho_{1}(x)=\psi^{\prime}(x) \quad\left(0 \leqslant x \leqslant \frac{1}{\sqrt{2}}\right) .
$$

Sia poi

$$
\varrho_{2}(x)=1, \quad\left(0 \leqslant x \leqslant \frac{1}{\sqrt{2}}\right) .
$$

Si ha $\varrho_{1}(x)=\operatorname{tg} \alpha_{0}\left(\operatorname{con} \pi / 4<\alpha_{0}<\pi / 2\right)$ oppure $\varrho_{1}(x)=\operatorname{tg} \gamma_{n}$ (con $\left.0<\gamma_{n}<\pi / 4\right)$, quindi è $\varrho_{1}(x) \leqslant \operatorname{tg} \alpha_{0}$; essendo $\varrho_{2}(x)=1$, è anche $\varrho_{2}(x)<\operatorname{tg} \alpha_{0}$.

Siano

$$
Y=g_{i}(X ; x, y) \quad(i=1,2)
$$

le funzioni definite dalle

$$
g_{i}(X ; x, y)=y+\int_{x}^{x} g_{i}(t) d t
$$

nel campo

$$
\Delta_{\infty}: 0 \leqslant X \leqslant \frac{1}{\sqrt{2}}, \quad 0 \leqslant x \leqslant \frac{1}{\sqrt{2}},-\infty<y<+\infty .
$$

E

$$
g_{1}(X ; x, y)=y-\psi(x)+\psi(X), \quad g_{2}(X ; x, y)=y-x+X,
$$


in particolare

$$
g_{2}(0 ; x, y)=y-x
$$

Siano inoltre $f_{1}(x), f_{2}(x)$ due funzioni quasi continue e integrabili in $(0,1 / \sqrt{2})$. Siano infine $\phi_{1}(y), \phi_{2}(y)$ due funzioni assolutamente continue in ogni intervallo $(-b, b)$ eon

$$
\phi_{\mathrm{s}}(y)=\sqrt{|y|}
$$

Consideriamo il sistema

$$
\left\{\begin{aligned}
z_{1}(x, y)+z_{2}(x, y) & =\phi_{1}\left(g_{1}(0 ; x, y)\right)+\phi_{2}\left(g_{1}(0 ; x, y)\right)+\int_{0}^{x} f_{1}(t) d t \\
z_{2}(x, y) & =\quad \phi_{2}\left(g_{2}(0 ; x, y)+\int_{0}^{x} f_{2}(t) d t .\right.
\end{aligned}\right.
$$

Il sistema (52) è un particolare sistema (10), per il quale valgono tutte le ipotesi dei nn. 1, 3 tranne la (7).

Dalla seconda equazione segue necessariamente

$$
z_{2}(x, y)=\sqrt{|y-x|}+\int_{0}^{x} f_{2}(t) d t
$$

nel campo

$$
D: \quad 0 \leqslant x \leqslant \frac{1}{\sqrt{2}},-b+x \operatorname{tg} \alpha_{0} \leqslant y \leqslant b-x \operatorname{tg} \alpha_{0} \quad\left(b \geqslant \frac{\operatorname{tg} \alpha_{0}}{\sqrt{2}}\right),
$$

dopodichè la prima delle $(52)$ determina $z_{1}(x, y)$.

E evidente che le funzioni $z_{1}(x, y), z_{2}(x, y)$ sono assolutamente continue rispetto a $y$, per ogni $x$ fissato, nell'intervallo $-b+x \operatorname{tg} \alpha_{0} \leqslant y \leqslant b-x \operatorname{tg} \alpha_{0}$, e, per ogni $y_{0}$ fissato in $(-b, b)$, assolutamente continue in $x$ sul segmento della retta $y=y_{0}$ appartenente a $D$ : infatti tali sono le funzioni $\phi_{i}\left(g_{1}(0 ; x, y)\right), \phi_{i}\left(g_{2}(0 ; x, y)\right),(i=1,2)$, come funzioni assolutamente continue di funzioni $g_{1}(0 ; x, y), g_{2}(0 ; x, y)$ monotone $\left.{ }^{20}\right)$ sia rispetto a $x$ che rispetto a $y$.

Assunte, nella $\left(52^{\prime}\right), X, Y$ come coordinate correnti, e considerata la funzione $z_{2}(X, Y)$ per $Y=g_{1}(X ; 0,0)$

$$
z_{2}\left(X, g_{1}(X ; 0,0)\right)
$$

(20) Essendo $\varrho_{1}(x)>0, \varrho_{2}(x)>0,(0 \leqslant x \leqslant 1 / \sqrt{2})$, le funzioni $g_{i}(X ; x, y), \quad(i=1,2)$, sono crescenti, oltre che rispetto a $y$ (come sempre si verifica in virtù del teorema di unicita citato in $\left({ }^{2 b i s}\right)$ ), anche rispetto a $X$, e decrescenti rispetto a $x$ (efr. $\S 1, n .2$ ). 
tale funzione non è assolutamente continua in $X$ : si dimostra infatti addirittura che non è a variazione limitata.

Considerato un punto $P(X, Y)$, utilizziamo la notazione $z_{2}(P)=z_{2}(X, Y)$.

Si ha (cfr. la figura, nella quale si intende sostituire le coordinate $X, Y$ alle coordinate $x, y$; cfr. anche nota $\left({ }^{19}\right)$, per il significato di $a_{n}$ )

$$
z_{2}\left(P_{2 n+1}\right)-z_{2}\left(P_{2 n+2}\right)=\sqrt{d_{n} \sqrt{2}}+\int_{X_{2 n+2}}^{X_{2 n+1}} f_{2}(t) d t, \quad(n=0,1, \ldots),
$$

dove si è osservato che in $P_{2 n+2}$ è $Y-X=0$ e in $P_{2 n+1}$ è $Y-X=d_{n} \sqrt{2}$.

Essendo

$$
d_{n}=\operatorname{tg} \alpha\left(\frac{1}{2 n+1}-\frac{1}{2 n+2}\right) \quad(n=0,1,2, \ldots)
$$

si ha anche

$$
d_{n}>\frac{\operatorname{tg} \alpha}{(2 n+2)^{2}} \quad(n=0,1,2, \ldots)
$$

Ne segue

$$
\sum_{n=0}^{r}\left|z_{2}\left(P_{2 n+1}\right)-z_{2}\left(P_{2 n+2}\right)\right|=\sum_{n=0}^{r}\left|\sqrt{\frac{\sqrt{2} \operatorname{tg} \alpha}{(2 n+1)(2 n+2)}}+\int_{X_{2 n+3}}^{X_{2 n+1}} f_{2}(t) d t\right|,
$$

e se, per semplificare, supponiamo $f_{2}(t) \geqslant 0$,

$$
\sum_{n=0}^{r}\left|z_{2}\left(P_{2 n+1}\right)-z_{2}\left(P_{2 n+2}\right)\right|>\sum_{n=0}^{r} \frac{\sqrt{\sqrt{2} \operatorname{tg} \alpha}}{2 n+2}=\sqrt{\sqrt{2} \operatorname{tg} \alpha}\left(\frac{1}{2}+\frac{1}{4}+\ldots+\frac{1}{2 r+2}\right),
$$

da cui subito segue che la funzione $z_{2}\left(X, g_{1}(X ; 0,0)\right)$ non è a variazione limitata.

OSSERVAZIONE. - Le caratteristiche del secondo sistema hanno in comune infiniti punti con le caratteristiche del primo sistema, senza avere archi in comune.

Ciò si verifica per esempio per le due caratteristiche

$$
Y=X, \quad Y=g_{1}(X ; 0,0) .
$$

10. - a) Lemma. - Siano $f_{1}(x, y), f_{2}(x, y)$ due funzioni definite nel campo

$$
D_{\infty}: \quad 0 \leqslant x \leqslant a_{0}, \quad-\infty<y<+\infty,
$$

le quali in corrispondenza ad ogni y siano quasi continue rispetto a $x$ in $\left(0, a_{0}\right)$; esistano due funzioni non negative, quasi continue e integrabili in $\left(0, a_{0}\right), \eta(x), \lambda(x)$, tali che in quasi tutto $\left(0, a_{0}\right)$ sia

$$
\left|f_{i}(x, y)\right| \leqslant \eta(x) \quad(i=1,2)
$$


per ogni y reale, $e$

$$
\left|f_{i}(x, y)-f_{i}(x, \bar{y})\right| \leqslant \lambda(x)|y-\bar{y}| \quad(i=1,2)
$$

per ogni coppia di valori reali $y, \bar{y}$.

Inoltre per quasi tutti gli $x$ di $\left(0, a_{0}\right)$ sia

$$
f_{1}(x, y) \leqslant f_{2}(x, y)
$$

per ogni $y$.

Considerate le equazioni differenziali

$$
\begin{aligned}
& y^{\prime}=f_{1}(x, y) \\
& y^{\prime}=f_{2}(x, y)
\end{aligned}
$$

e indicati con $y_{1}(x), y_{2}(x)$ due integrali rispettivamente della prima e seconda equazione, soddisfacenti le

$$
y_{1}\left(x_{0}\right)=y_{2}\left(x_{0}\right)=y_{0}
$$

dove $x_{0}$ è un punto dell'intervallo $\left(0, a_{0}\right)$, valgono le

$$
\begin{cases}y_{1}(x) \leqslant y_{2}(x) & \text { per } x_{0}<x \leqslant a_{0} \\ y_{1}(x) \geqslant y_{2}(x) & \text { per } 0 \leqslant x<x_{0}\end{cases}
$$

se in particolare per quasi tutti gli $x$ di $\left(0, a_{0}\right)$ vale la

$$
f_{1}(x, y)<f_{2}(x, y)
$$

per ogni $y$, si ha

$$
\begin{cases}y_{1}(x)<y_{2}(x) & \text { per } x_{0}<x \leqslant a_{0} \\ y_{1}(x)>y_{2}(x) & \text { per } 0 \leqslant x<x_{0} .\end{cases}
$$

Ne segue che, nell'ipotesi $\left(55^{\prime}\right)$, due curve integrali delle equazioni $\left(56_{1}\right),\left(56_{2}\right)$ rispettivamente, le quali abbiano in comune un punto, non hanno altri punti in comune.

Ossertazione I. - Se le funzioni $f_{1}(x, y), f_{2}(x, y)$ sono continue, la proprietà è immediata.

Osservazione II. - La proprietà è evidente se è $f_{1}(x, y) \leqslant 0, f_{2}(x, y) \geqslant 0$.

b) In virtù dei teoremi di esistenza e unicità di Carathéodory (cfr. 1.c. in $\left(^{2}\right)$ ) esiste, ed è unica, la funzione $y_{i}(x)$, assolutamente continua in $\left(0, a_{0}\right)$, che soddisfa quasi ovunque in $\left(0, a_{0}\right)$ l'equazione $\left(56_{i}\right)$ e la condizione

$$
y_{i}\left(x_{0}\right)=y_{0}, \quad(i=1,2)
$$


in quasi tutto $\left(0, a_{0}\right)$ è dunque

$$
\begin{aligned}
& y_{1}^{\prime}(x)=f_{1}\left(x, y_{1}(x)\right) \\
& y_{2}^{\prime}(x)=f_{2}\left(x, y_{2}(x)\right) .
\end{aligned}
$$

Dalle $(58)$ segue in quasi tutto $\left(0, a_{0}\right)$

$$
\frac{d\left(y_{2}(x)-y_{1}(x)\right)}{d x}=f_{2}\left(x, y_{2}(x)\right)-f_{1}\left(x, y_{1}(x)\right)
$$

e anche

$$
\frac{d\left(y_{2}(x)-y_{1}(x)\right)}{d x}=f_{2}\left(x, y_{2}(x)\right)-f_{2}\left(x, y_{1}(x)\right)+f_{2}\left(x, y_{1}(x)\right)-f_{1}\left(x, y_{1}(x)\right)
$$

Definiamo in $\left(0, a_{0}\right)$ la funzione $H(x)$ nel modo che segue: in quei punti di $\left(0, a_{0}\right)$ nei quali è $y_{1}(x) \neq y_{2}(x)$ poniamo

$$
H(x)=\frac{f_{2}\left(x, y_{2}(x)\right)-f_{2}\left(x, y_{1}(x)\right)}{y_{2}(x)-y_{1}(x)},
$$

nei rimanenti punti

$$
H(x)=0 .
$$

Osserviamo che $H(x)$ risulta quasi continua in $\left(0, a_{0}\right)$, inoltre, in virtù delle $(54)$, in quasi tutto $\left(0, a_{0}\right) \grave{\mathrm{e}}$

$$
|H(x)| \leqslant \lambda(x) .
$$

La (59) si scrive allora

$$
\frac{d\left(y_{2}(x)-y_{1}(x)\right)}{d x}=H(x)\left(y_{2}(x)-y_{1}(x)\right)+f_{2}\left(x, y_{1}(x)\right)-f_{1}\left(x, y_{1}(x)\right)
$$

la $\left(59^{\prime}\right)$ vale per quasi tutti gli $x$ di $\left(0, a_{0}\right)$.

Consideriamo ora l'equazione differenziale lineare

$$
y^{\prime}=H(x) y+f_{2}\left(x_{1} y_{1}(x)\right)-f_{1}\left(x_{1} y_{1}(x)\right) .
$$

Essa ammette come integrale la funzione

$$
y_{2}(x)-y_{1}(x),
$$

la quale, per $x=x_{0}$, assume valore nullo. 
Esiste un solo $\left({ }^{21}\right)$ integrale $Y(x)$ della $(61)$ soddisfacente la condizione

$$
Y\left(x_{0}\right)=0
$$

esso ha la forma $\left({ }^{22}\right)$

$$
Y(x)=\int_{x_{0}}^{\tilde{x}}\left[f_{2}\left(X, y_{1}(X)\right)-f_{1}\left(X, y_{1}(X)\right)\right] \exp \left[\int_{X}^{x} H(t) d t\right] d X
$$

Pertanto si ha $y_{2}(x)-y_{1}(x)=Y(x)$ in tutto $\left(0, a_{0}\right)$, vale a dire

$$
y_{2}(x)-y_{1}(x)=\int_{x_{0}}^{x}\left[f_{2}\left(X, y_{1}(X)\right)-f_{1}\left(X, y_{1}(X)\right)\right] \exp \left[\int_{X}^{x} H(t) d t\right] d X,
$$

da cui, tenuto conto delle (55), seguono le (57); se valgono le (55'), ne seguono le $\left(57^{\prime}\right)$.

(21) Consideriamo l'equazione lineare $y^{\prime}=H(x) y+K(x)$, con $H(x), K(x)$ quasi continue e integrabili in $\left(0, a_{0}\right)$. Se $Y(x), Y^{*}(x)$ sono due integrali di tale equazione, soddisfacenti la condizione $Y\left(x_{0}\right)=Y^{*}\left(x_{0}\right)=Y_{0}$, in $\left(0, a_{0}\right)$ valgono le

da cui

$$
Y(x)=Y_{0}+\int_{x_{0}}^{x}[H(X) \cdot Y(X)+K(X)] d X, \quad Y^{*}(x)=Y_{0}+\int_{x_{0}}^{x}\left[H(x) Y^{*}(X)+K(X)\right] d X,
$$

$$
Y(X)-Y^{*}(X)=\int_{\infty_{0}}^{x} H(X)\left[Y(X)-Y^{*}(X)\right] d X
$$

Un'immediata applicazione del lemma di Gronwall generalizzato (cfr. 1.c. in ( $\left.{ }^{15}\right)$ ) prova che è $\bar{Y}(x)=Y^{*}(x)$ in tutto $\left(0, a_{0}\right)$.

$\left({ }^{22}\right)$ Più in generale, l'integrale dell'equazione lineare $y^{\prime}=H(x) y+K(x)$, soddisfacente la condizione $y\left(x_{0}\right)=y_{0} \check{\mathrm{e}}$

$$
y=y_{0} \exp \left[\int_{x_{0}}^{x} H(X) d X\right]+\int_{x_{0}}^{x} K(X) \exp \left[\int_{X}^{x} H(t) d t\right] d X .
$$

